\title{
FUS driven circCNOT6L biogenesis in mouse and human spermatozoa supports zygote development
}

\author{
Teresa Chioccarelli ${ }^{1}$. Geppino Falco ${ }^{2,3}$. Donato Cappetta ${ }^{1}$ - Antonella De Angelis ${ }^{1}$ - Luca Roberto ${ }^{3}$ Martina Addeo ${ }^{2}$. \\ Marco Ragusa ${ }^{4}$. Davide Barbagallo ${ }^{4}$. Liberato Berrino ${ }^{1}$. Michele Purrello ${ }^{4}$. Concetta Ambrosino ${ }^{3,5}$. Gilda Cobellis ${ }^{1}$. \\ Riccardo Pierantoni ${ }^{1} \cdot$ Rosanna Chianese ${ }^{1}\left[{ }^{(0)} \cdot\right.$ Francesco Manfrevola $^{1}$
}

Received: 22 June 2021 / Revised: 10 November 2021 / Accepted: 19 November 2021 / Published online: 22 December 2021

(c) The Author(s) 2021

\begin{abstract}
Circular RNA (circRNA) biogenesis requires a backsplicing reaction, promoted by inverted repeats in cis-flanking sequences and trans factors, such as RNA-binding proteins (RBPs). Among these, FUS plays a key role. During spermatogenesis and sperm maturation along the epididymis such a molecular mechanism has been poorly explored. With this in mind, we chose circCNOT6L as a study case and wild-type (WT) as well as cannabinoid receptor type-1 knock-out $\left(\mathrm{CbI}^{-/-}\right)$male mice as animal models to analyze backsplicing mechanisms. Our results suggest that spermatozoa (SPZ) have an endogenous skill to circularize mRNAs, choosing FUS as modulator of backsplicing and under CB1 stimulation. A physical interaction between FUS and CNOT6L as well as a cooperation among FUS, RNA Polymerase II (RNApol2) and Quaking (QKI) take place in SPZ. Finally, to gain insight into FUS involvement in circCNOT6L biogenesis, FUS expression was reduced through RNA interference approach. Paternal transmission of FUS and CNOT6L to oocytes during fertilization was then assessed by using murine unfertilized oocytes (NF), one-cell zygotes (F) and murine oocytes undergoing parthenogenetic activation (PA) to exclude a maternal contribution. The role of circCNOT6L as an active regulator of zygote transition toward the 2-cell-like state was suggested using the Embryonic Stem Cell (ESC) system. Intriguingly, human SPZ exactly mirror murine SPZ.
\end{abstract}

Keywords Backsplicing $\cdot$ Cannabinoid receptor I $\cdot$ circRNAs $\cdot$ Sperm maturation $\cdot$ Embryo development

\section{Introduction}

Mammalian cells retain thousands of circular RNAs (circRNAs) with tissue-specific and cell type expression patterns [1-4].

Rosanna Chianese

rosanna.chianese@unicampania.it

1 Dipartimento di Medicina Sperimentale, Sez. Bottazzi, Università degli Studi della Campania "L. Vanvitelli”, Via Costantinopoli 16, 80138 Napoli, Italy

2 Dipartimento di Biologia, Università di Napoli "Federico II", Napoli, Italy

3 Istituto di Ricerche Genetiche Gaetano Salvatore, Biogem scarl, Ariano Irpino, Avellino, Italy

4 Dipartimento di Scienze Biomediche e Biotecnologiche, Università di Catania, Via Santa Sofia 97, 95123 Catania, Italy

5 Dipartimento di Scienze e Tecnologie, Università del Sannio, Benevento, Italy
The molecular mechanism promoting circRNA biogenesis requires a backsplicing reaction that-unlike canonical splicing-covalently binds a downstream splice donor site reversely with an upstream splice acceptor site [5]. The circularization of exonic circRNAs is promoted by inverted repeats in cis-flanking sequences [6], as well as by trans factors, such as RNA-binding proteins (RBPs) which have conserved binding sites in the flanking introns $[7,8]$. The Fused protein in Sarcoma (FUS) gene plays a crucial role in circRNA biogenesis during Embryonic Stem Cell (ESC) differentiation [9]. Other RBPs have also been implicated [4, 10, 11]. Conversely, Adenosine (A) to Inosine (I) editing by ADAR deaminase, proposed to destabilize the base-pairing necessary for backsplicing reaction, perturbs circRNA biogenesis [12]. $N^{6}$-methyladenosine $\left(\mathrm{m}^{6} \mathrm{~A}\right)$ has recently been suggested to promote circRNA accumulation in late spermiogenesis to compensate for massive degradation of linear mRNAs, with several aspects of the involved molecular machinery to be unveiled [13]. 
In this scenario, circRNA biogenesis and degradation during spermatogenesis and sperm maturation along the epididymis have been poorly explored. To this scope, we used circCNOT6L as a study case considering that it has been recently discovered in human spermatozoa (SPZ), localized in sperm head, with a potential role in embryo development [3]. Its linear counterpart (CNOT6L-mRNA) is a key component of the eukaryotic de-adenylase complex CCR4-NOT [14], implicated in the maternal mRNA decay pathway, during the oocyte-to-embryo transition [15]. Although genetic deletion of Cnot $6 L$ yields viable mice, females in the offspring are severely sub-fertile [16]: eggs develop slower and, frequently, arrest at prometaphase [17]. In addition, the over-translation of maternal undegraded mRNAs causes microtubule-chromosome disorganization that leads to a spindle assembly checkpoint and meiotic cell cycle arrest [16].

The biogenesis of circCNOT6L as well as FUS and ADAR levels in testis and sperm were explored in cannabinoid receptor type-1 (CB1) knock-out $\left(\mathrm{Cbl}^{-/-}\right)$male mice, chosen for their altered reproductive phenotype in comparison to wildtype (WT) animals [18-23]. Our results led us to hypothesize CB1 involvement in backsplicing that we confirmed by in vitro assays.

Focusing on sperm cells, we evaluated their ability to circularize mRNAs during their transit along the epididymis, choosing FUS protein as a key modulator of backsplicing under CB1 stimulation. Therefore FUS protein localization was assessed by immunofluorescence; then the physical interaction between FUS and CNOT6L and the cooperation among FUS, RNA Polymerase II (RNApol2) and Quaking (QKI) were demonstrated by RNA immunoprecipitation assay (RIP) and immunoprecipitation assay (IP), respectively. Finally, to gain insight into FUS involvement in circCNOT6L biogenesis, FUS expression was reduced through an RNA interference approach.

Encouraged by a bioinformatic prediction according to which several downstream mRNA targets of circCNOT6L are associated with embryo development, we evaluated the paternal transmission of FUS and CNOT6L to oocytes, during fertilization, verified the only involvement of sperm in such a mechanism using parthenogenetically activated oocytes and defined the role of circCNOT6L during the early phases of zygote development using a 2-cell-like ESC system.

Intriguingly, what was observed in mouse SPZ was also confirmed in humans.

\section{Material and methods}

\section{Experimental animals}

WT male mice or males carrying a $C b 1$ null mutation [24] were used in this study. Heterozygous mice were bred on a CD1 background (Charles River Laboratory, Lecco, Italy) before generating male mice (WT, $\mathrm{Cbl}^{-/-}$). Adult males (4-8 months) under anesthesia were sacrificed by cardiac perfusion with PBS (pH 7.6) to clean peripheral tissues (testes, epididymides and SPZ) from blood contaminants. In detail, testes were rapidly removed and stored at $-80{ }^{\circ} \mathrm{C}$, while epididymides were dissected and used to collect total epididymal SPZ or SPZ from caput (caput SPZ) and cauda (cauda SPZ) regions, and relative epididymal tissues (SPZ-deprived epididymis), depending on the experimental procedure, as described below.

\section{Human sperm collection}

Human semen samples were obtained from normozoospermic volunteer donors $(n=5)$ through masturbation after 3-5 days of sexual abstinence and collected in sterile sample containers, which were delivered to the laboratory within $1 \mathrm{~h}$ after ejaculation. The sperm samples were allowed to liquefy for $30 \mathrm{~min}$ at $37{ }^{\circ} \mathrm{C}$ and were then purified on a discontinuous density gradient. In particular, using a $40 \%$ and $80 \%$ discontinuous PureCeption (Cooper Surgical, Trumbull, CT, United States) gradient, we were able to purify viable and motile SPZ from the base of the $80 \%$ PureCeption fraction ("A-SPZ" of good quality) and abnormal SPZ from 40\% PureCeption fraction ("B-SPZ" of poor quality). An aliquot of all sperm samples was used to assess sperm vitality by Trypan blue staining (Trypan Blue, $0.4 \%$ Solution, 17-942E Lonza). The analysis of live SPZ was performed under a light microscope by counting the percentage of live/total SPZ.

A- and B-SPZ fractions were then treated with Somatic Cell Lysis Buffer (SCLB) consisting of $0.1 \%$ SDS, $0.5 \%$ Triton X-100 in DEPC- $\mathrm{H}_{2} \mathrm{O}$, to eliminate possible contamination by somatic cells. In brief, sperm pellets were incubated with an appropriate volume of SCLB on ice for $30 \mathrm{~min}$. After lysis, SPZ were centrifuged at $300 \times g$ for $15 \mathrm{~min}$ at $4{ }^{\circ} \mathrm{C}$ and then washed twice with sperm washing medium (HTF-IrvineScientific ${ }^{\circledR}$ ). Following SCLB treatment and microscope examination carried out to verify the elimination of somatic cells, an aliquot of A- and B-SPZ samples was used to re-evaluate the number of live SPZ under a light microscope to exclude effects on sperm vitality and concentration induced by the technical procedure (data not shown). A- and B-SPZ pellets were stored at $-80{ }^{\circ} \mathrm{C}$ for RNA or protein extraction and dried on slides and finally stored at $-20{ }^{\circ} \mathrm{C}$ for immunofluorescence analysis. 


\section{Sperm collection from mouse caput and cauda epididymis}

Total epididymis (from $n=3 \mathrm{WT}$ and $n=3 \mathrm{Cbl}^{-/-}$) or caput/cauda epididymis (from $n=3 \mathrm{WT}$ ) were separately immersed in PBS (pH 7.6) and cut to let SPZ flow out from the ducts. Samples of total and/or caput and cauda SPZ were then filtered throughout cheesecloth to eliminate fragments of epididymal tissue and centrifuged at $1500 \times \mathrm{g}$ for $30 \mathrm{~min}$ at $4{ }^{\circ} \mathrm{C}$. The epididymal fragments were separately frozen as pieces of total or caput and cauda epididymis deprived of sperm cells.

After centrifugation, the SPZ pellet was incubated on ice for $30 \mathrm{~min}$ with SCLB $(0.1 \%$ SDS, $0.5 \%$ Triton X-100 in DEPC- $\mathrm{H}_{2} \mathrm{O}$ ) to eliminate possible contamination by somatic cells. After lysis, SPZ were centrifuged at $800 \times g$ for $15 \mathrm{~min}$ at $4{ }^{\circ} \mathrm{C}$ and then washed twice with PBS. Aliquots of total epididymal SPZ from WT and $\mathrm{Cbl}^{-/-}$or caput and cauda SPZ from WT mice were both stored at $-80{ }^{\circ} \mathrm{C}$ for RNA or protein extraction and dried on slides to be finally stored at $-20{ }^{\circ} \mathrm{C}$ for immunofluorescence analysis.

\section{ACEA in vitro treatment of mouse testis and SPZ, and human SPZ}

ACEA (arachidonyl-2-chloroethylamide), a selective CB1 receptor agonist, was obtained from Sigma-Aldrich (A9719; Milan, Italy). The drug was dissolved in dimethylsulfoxide (DMSO) according to the manufacturer's instructions.

WT testes with a feeble notch in tunica albuginea $(n=3$ for experimental group) were incubated in PBS $(6 \mathrm{ml})$ for $90 \mathrm{~min}$ at room temperature (RT), with vehicle $(0.005 \%$ DMSO; control group, CTRL) or with ACEA at $0.1 \mu \mathrm{M}$, $1 \mu \mathrm{M}$ and $10 \mu \mathrm{M}$. After treatment the testes were kept at $-80{ }^{\circ} \mathrm{C}$.

Caput SPZ from WT mice or human B-SPZ fraction from normozoospermic volunteers were purified as described above and incubated in PBS $(1 \mathrm{ml})$ for $30 \mathrm{~min}$ at $37{ }^{\circ} \mathrm{C}$ with vehicle $(0.005 \%$ DMSO; CTRL) or with ACEA at $1 \mu \mathrm{M}$. After treatment SPZ were centrifuged at $1500 \times g$ for $20 \mathrm{~min}$ at $4{ }^{\circ} \mathrm{C}$ and washed twice with PBS. The sperm pellet was stored at $-80{ }^{\circ} \mathrm{C}$.

\section{Immunofluorescence analysis on mouse and human SPZ}

Mouse and human SPZ dried on slides as above reported were fixed in 4\% paraformaldehyde (sc-281692; Santa Cruz Biotechnology, Heidelberg, Germany) for $20 \mathrm{~min}$ at RT and then permeabilized with $0.1 \%$ Triton $\mathrm{X}-100$ (X100; Sigma-Aldrich, Milano, Italy). After permeabilization, blocking was conducted with $10 \%$ of donkey serum (ab7475; Abcam, Cambridge, UK) for $30 \mathrm{~min}$ at RT and the cells were then incubated with anti-FUS antibody $(\mathrm{Ab})$ (PA5-52610; Invitrogen, Milano, Italy), overnight at $4{ }^{\circ} \mathrm{C}$. Following three washes in Dulbecco's PBS (DPBS, 1X), a fluorescein isothiocyanate (FITC) conjugated $\mathrm{Ab}$ was used (711-095-152; Jackson ImmunoResearch, Cambridge, UK) for $1 \mathrm{~h}$ at $37^{\circ} \mathrm{C}$. Nuclei were labeled with DAPI (D9542; Sigma-Aldrich, Milano, Italy) and slides were analyzed with a Zeiss LSM700 confocal microscope.

\section{Vesicle shuttle in vitro experiment}

Caput and cauda epididymis (from $n=8 \mathrm{WT}$ ) were separately pulled in PBS (pH 7.6) and cut to let SPZ flow out from the ducts. Caput and cauda SPZ samples were then filtered throughout cheesecloth to eliminate fragments of epididymal tissue and centrifuged at $1500 \times g$ for $30 \mathrm{~min}$ at $4{ }^{\circ} \mathrm{C}$. The resulting fluid was further clarified via centrifugation $\left(16,000 \times g\right.$ for $30 \mathrm{~min}$ at $\left.4{ }^{\circ} \mathrm{C}\right)$ with the supernatant yielding the Epididymal Luminal Fluid (ELF) [25]. ELF purity was checked for possible sperm contaminations under a light microscope (Leica Microsystems Inc., Milano, Italy). The SPZ pellet was obtained as described above; both the SPZ pellet and ELF were used for in vitro treatments as follows.

For each experimental group, $10 \times 10^{6}$ washed SPZ from caput epididymis were incubated for $30 \mathrm{~min}$ at $37{ }^{\circ} \mathrm{C}$ in $1 \mathrm{ml}$ of: (1) PBS (CTRL group); (2) Caput ELF; (3) Cauda $\mathrm{ELF}$; (4) Cauda ELF pre-treated for $2 \mathrm{~h}$ at $37^{\circ} \mathrm{C}$ with antiCD9 Ab (sc-13118; Santa Cruz Biotechnologies, Heidelberg, Germany) at concentration of 10-1000 ng/ml [26]; (5) Cauda ELF in presence of ACEA $1 \mu \mathrm{M}$.

After treatment SPZ were centrifuged at $1500 \times g$ for $20 \mathrm{~min}$ at $4{ }^{\circ} \mathrm{C}$ and washed twice with PBS. The sperm pellet was kept at $-80^{\circ} \mathrm{C}$.

\section{Mouse Zygote Manipulation}

Murine one-cell zygotes (F) were obtained as reported by Ragusa et al. [27]. In brief, for mature oocyte collection, natural cycling CD1 females $(n=12)$, in the estrus phase, were mated with fertile $(n=6)$ or vasectomized/sterile $(n=6)$ CD1 males, following the scheme: 1 male with 1 female. Pregnant female mice at 0.5 day post coituum $(d p c)$ were euthanized by cervical dislocation and the uterus was explanted. Then, an uterine horn was laid in a drop of Hyaluronidase from the bovine testes (H4272; Sigma-Aldrich, Milano, Italy) in M16 medium (M7292; Sigma-Aldrich, Milano, Italy) $1 \mathrm{mg} / \mathrm{ml}$, and the fertilization ampoule was broken to release embryos and the cells surrounding them. Then, oocytes were collected in a drop of PBS to wash them from blood residues. The F obtained from CD1 females mated with fertile males, were selected by stereomicroscope observation, excluding not fertilized oocytes. 
With the aim of collecting mature not fertilized oocytes (NF), we induced pseudopregnancy in females, by mating them with vasectomized/sterile males, to allow them to behave hormonally as pregnant [28]. With this approach, we selected by stereomicroscope observation the NF-in metaphase II (MII) stage - that extruded the first polar body (PB), comparably to F zygotes.

Six pools (1 pool/female), each containing $10 \mathrm{~F}$ or 10 NF, were used for expression analysis. For RNA and protein extraction, each experimental oocyte pool (F and NF) was subjected to thermolysis as described by Di Pietro et al. [29]: the samples were resuspended in $10 \mu \mathrm{l}$ of RNase-free water and incubated for $1 \mathrm{~min}$ at $100{ }^{\circ} \mathrm{C}$, put in ice for $1 \mathrm{~min}$ and, finally, vortexed for $30 \mathrm{~s}$. Released RNA and proteins were analyzed by qRT-PCR and western blot, respectively, as described below.

\section{Parthenogenetic activation of murine oocytes}

Parthenogenetic activation of murine oocytes (PA) was performed as previously reported by Kaufman [30]. Briefly, CD1 female mice $(n=6)$ were injected with 5 International Units (IU) of pregnant mare serum gonadotropin (PMSG) and $5 \mathrm{IU}$ of human chorionic gonadotropin (hCG). After 16-18 h, female mice were sacrificed. The oocytes, surrounded by cumulus cells, were recovered and then released into freshly prepared $7 \%$ ethanol in DPBS solution, in a $3 \mathrm{~cm}$ sterile tissue culture dish, for $5 \mathrm{~min}$ at RT. After three washes with DPBS and two washes with M2 medium (M7167, Merck, Germany), the cumulus masses were transferred to single drops of M16 medium (MR-016-D, Merck, Germany), covered by paraffin oil and incubated at $37{ }^{\circ} \mathrm{C}$ for $5 \mathrm{~h}$. Cumulus cells were then removed by Hyaluronidase (final concentration of $0.5-1 \mathrm{mg} / \mathrm{mL}$ ), and the activated oocytes were classified under a phase contrast microscope. Finally, the PA — in metaphase II (MII) stage - that extruded the $\mathrm{PB}$, comparably to NF and $\mathrm{F}$ zygotes, were transferred to single drops of M16 medium under paraffin oil and stored.

\section{ESC culture and Retinoic Acid treatment}

Generation of E14tg $2^{\text {pcDNA3_prZScan4_LNGFR }}$ was previously described [31]. The cells were cultured on gelatin-coated dishes in ES complete medium: GMEM (Sigma-Aldrich, Milano, Italy) supplemented with $15 \%$ FBS (GE Healthcare, Milano, Italy), L-glutamine $2 \mathrm{mM}$ (Gibco, Dublin, Ireland), sodium pyruvate $1 \mathrm{mM}$ (Gibco, Dublin, Ireland), MEM amino acids $1 \mathrm{X}$ (Gibco, Dublin, Ireland), penicillin/streptomycin $100 \mathrm{U} / \mathrm{ml}$ (Gibco, Dublin, Ireland), 2( $\beta)$ mercaptoethanol $0.1 \mathrm{mM}$ (Gibco, Dublin, Ireland), LIF $1000 \mathrm{U} / \mathrm{ml}$ (Millipore, Burlington, United States), and Geneticin $^{\text {(TM) }} 137.5 \mu \mathrm{g} / \mathrm{ml}$ (Gibco, Dublin, Ireland). The medium was changed daily and cells were routinely split every 2-3 days and incubated at $37{ }^{\circ} \mathrm{C}$ in $5 \% \mathrm{CO}_{2}$ For Retinoic Acid (RA) treatment, the cells were trypsinized and plated on gelatin-coated dishes in ES complete regular medium (RM) or ES complete medium supplemented with $1.5 \mu \mathrm{M}$ all-trans-RA (Sigma-Aldrich, Milano, Italy) (RA). After $72 \mathrm{~h}$, the cells were washed with ice-cold PBS (1X), trypsinized into a single cell suspension, and incubated with MACSelect ${ }^{(\mathrm{TM})}$ LNGFR MicroBeads (Miltenyi Biotec, Bologna, Italy) in PBS (1X) supplemented with $5 \mathrm{mM}$ EDTA, and $0.5 \%$ BSA (PBE Buffer) at $4{ }^{\circ} \mathrm{C}$ for $20 \mathrm{~min}$. Magnetically labeled cells were then isolated using the AutoMACS Pro Separator (Miltenyi Biotec, Bologna, Italy) with the "posseld2" program and prZscan4_LNGFR positive and negative fractions from RM and RA treatment homogeneously collected, according to the manufacturer's protocol.

\section{RNA interference}

For small interfering RNA (siRNA)-mediated knockdown of Fus mRNA, E14tg2 $2^{\text {pcDNA3_prZScan4_LNGFR }}$ stable cell line was thawed and cultured for 3 days in RM medium. Subsequently, the cells were reverse transfected with 5, 10, 25 and $50 \mathrm{nM}$ of Fus siGENOME SMART pool siRNA or siGENOME Non-Targeting as scramble control (siScr) (Dharmacon, Inc., United States). Briefly, siFus and siScr, at the selected concentrations, were complexed with DharmaFECT1 transfection reagent, according to the manufacturer's procedures. Then siRNA:DharmaFECT complex was added to 12 -well plates and incubated for $30 \mathrm{~min}$ at RT. Finally, $1.4 \times 10^{5}$ cells were plated in each well containing the selected siRNA concentration, in antibiotic-free cell culture medium. The cells were harvested after 48 or $72 \mathrm{~h}$ from siRNA transfection and used for total RNA or cell lysates preparation, as reported below.

\section{Total RNA preparation}

Total RNA was extracted from murine tissues, murine sperm cells, human sperm cells and murine E14tg $2^{\text {pcDNA3 }}$ prZScan4_LNGFR cells transfected with Fus siRNA or siScr, using Trizol Reagent (Invitrogen Life Technologies, Paisley, UK) following the manufacturer's instructions. In brief, samples were homogenized in Trizol Reagent $(1 \mathrm{ml}$ Trizol Reagent/mg tissue or $5-10 \times 10^{6}$ sperm cells); after homogenization, samples were incubated for $5 \mathrm{~min}$ at $20{ }^{\circ} \mathrm{C}$ to allow the complete dissociation of nucleoprotein complexes. Then $0.2 \mathrm{ml}$ chloroform $/ \mathrm{ml}$ Trizol Reagent was added and the sample centrifuged at $12,000 \times g$ for $15 \mathrm{~min}$ at $4{ }^{\circ} \mathrm{C}$. The aqueous phase was transferred to a fresh tube and total RNA was precipitated by mixing with isopropyl alcohol $(0.5 \mathrm{ml} / \mathrm{ml}$ Trizol Reagent $)$ and $1 \mu \mathrm{lgly}$ cogen $(20 \mathrm{mg} / \mathrm{ml})$ to promote the precipitation of small size RNAs. After centrifugation at $12,000 \times g$ for $10 \mathrm{~min}$ 
at $4{ }^{\circ} \mathrm{C}$, the RNA pellet was washed with $75 \%$ ethanol, centrifuged at $7500 \times g$ for $10 \mathrm{~min}$ at $4{ }^{\circ} \mathrm{C}$ and dissolved in an appropriate volume of DEPC-treated water. The quantity (ng/ $\mu \mathrm{l})$ and purity (260/280 and 260/230 ratios) of total RNAs were assessed with a NanoDrop 2000 spectrophotometer (Thermo, Waltham, MA, United States). To remove potential contamination of genomic DNA, RNA aliquots $(10 \mu \mathrm{g})$ were treated with 2U DNase I (RNase-free DNase I, Ambion, Thermo Fisher Scientific, Massachusetts, United States). The RNAs were then preserved at $-80{ }^{\circ} \mathrm{C}$ until the next step.

\section{RNA expression analysis by One-Step Evagreen qRT-PCR}

We investigated the expression of circCNOT6L and its linear counterpart (CNOT6L-mRNA) through One-Step Evagreen qRT-PCR reaction using a kit containing qRT-PCR enzyme mix and an Evagreen qPCR Mastermix (Applied Biological Materials Inc., Ferndale, WA, United States), according to the manufacturer's instructions. All reactions were performed using $50 \mathrm{ng}$ of total RNA on a CFX-96 Real Time PCR System (Biorad, Milano, Italy). Assays were carried out in triplicates and included a melting curve analysis for which all samples displayed single peaks for each primer pairs. A negative control, without RNA, was also included. RNA expression was evaluated through CFX Manager software (Biorad, Milano, Italy). Normalization was performed using Actin or GAPDH (glyceraldehyde 3-phosphate dehydrogenase) as housekeeping genes, for mouse and human samples, respectively. Normalized fold expression (nfe) of circCNOT6L and CNOT6L mRNA was calculated by applying the $2^{-\Delta \Delta \mathrm{Ct}}$ method. Results were expressed as mean value of nfe \pm SEM.

\section{PCR primer design}

Specific primers for circCNOT6L and CNOT6L-mRNA were designed through the online tool Primer-BLAST (http://www.ncbi.nlm.nih.gov/tools/primer-blast/). To make primers specific for the circular isoforms, we designed primers spanning the backsplicing junctions. We also designed specific primers for the housekeeping genes used for normalization: Actin or GAPDH, for mouse and human samples, respectively. All primer sequences are shown in Table 1.

\section{RNA binding protein immunoprecipitation assay (RIP)}

For RIP assay, $1 \times 10^{7}$ of mice caput SPZ or human B-SPZ were lysed in $500 \mu$ of RIP lysis buffer $(50 \mathrm{mM}$ Tris- $\mathrm{HCl}$
Table 1 Primers sequence and annealing temperatures

\begin{tabular}{|c|c|c|}
\hline Gene primers & Sequences $5^{\prime}-3^{\prime}$ & $\operatorname{Tm}\left({ }^{\circ} \mathrm{C}\right)$ \\
\hline Mти-circCNOT6LS & $\begin{array}{l}\text { ATTTACGGGTGTTGCCTT } \\
\text { ATGA }\end{array}$ & 56 \\
\hline Мти-circCNOT6L AS & $\begin{array}{l}\text { TGCGAGGATCTGGAGGAT } \\
\text { CA }\end{array}$ & \\
\hline Mmи-CNOT6L-mRNA $S$ & $\begin{array}{l}\text { CGTCCTGGGCATTAAACT } \\
\text { GG }\end{array}$ & 56 \\
\hline Мти-CNOT6L-mRNA AS & $\begin{array}{l}\text { CCACGATCCTTCAATGCT } \\
\text { GG }\end{array}$ & \\
\hline Мти-сіrcACTB $S$ & $\begin{array}{l}\text { GGCTGTATTCCCCTCCAT } \\
\text { CG }\end{array}$ & 55 \\
\hline Мти-сіrсACTB $A S$ & $\begin{array}{l}\text { CCAGTTGGTAACAATGCC } \\
\text { ATGT }\end{array}$ & \\
\hline Мти-FUS S & $\begin{array}{l}\text { GGTGGTGGAGGCAACTAT } \\
\text { GG }\end{array}$ & 56 \\
\hline Mmи-FUS AS & $\begin{array}{l}\text { GTCACTTCCGCCCATGCC } \\
\text { GC }\end{array}$ & \\
\hline Hsa-circCNOT6LS & $\begin{array}{l}\text { GCCTTATGAACTTGGTCG } \\
\text { GCT }\end{array}$ & 56 \\
\hline Hsa-circCNOT6L AS & $\begin{array}{l}\text { TTCTGCGAGGATCTGGAG } \\
\text { GAT }\end{array}$ & \\
\hline Hsa-CNOT6L-mRNA S & $\begin{array}{l}\text { TCGCAGTTCATCCAGAGC } \\
\text { AG }\end{array}$ & 54 \\
\hline Hsa- CNOT6L-mRNA AS & $\begin{array}{l}\text { ACGGCAGAATTTGGTCTC } \\
\text { GT }\end{array}$ & \\
\hline Hsa-circGAPDH S & $\begin{array}{l}\text { TGCACCACCAACTGCTTA } \\
\text { GC }\end{array}$ & 58 \\
\hline Hsa-circGAPDH AS & $\begin{array}{l}\text { GGCATGGACTGTGGTCAT } \\
\text { GAG }\end{array}$ & \\
\hline
\end{tabular}

pH 7.4; $150 \mathrm{mM} \mathrm{NaCl} ; 5$ mM EDTA; $1 \%$ NP-40; 0.1\% SDS) supplemented with protease inhibitors $(10 \mu \mathrm{g} / \mathrm{ml}$ of leupeptin, aprotinin, pepstatin A, chymostatin, and $5 \mu \mathrm{g} /$ $\mathrm{ml}$ of TPCK) and RNase inhibitors $(100 \mathrm{U} / \mathrm{ml})$. An aliquot of total lysate was removed from each sample for following input analysis. Equal concentration of lysate was incubated with $5 \mu \mathrm{g}$ of FUS Ab (PA5-52610; Invitrogen, Milano, Italy) or IgG (12370; Sigma-Aldrich, Milano, Italy) under rotary agitation at $4{ }^{\circ} \mathrm{C}$ overnight. Afterwards $60 \mu \mathrm{l}$ of slurry of Protein A/G PLUS Agarose Beads (sc2003; Santa Cruz Biotechnology, Heidelberg, Germany) was added to each sample and incubated at $4{ }^{\circ} \mathrm{C}$ for $4 \mathrm{~h}$. Then pellets were washed four times with cold TBS $\mathrm{pH}$ 7.6 at $3000 \times g$ for $5 \mathrm{~min}$ at $4{ }^{\circ} \mathrm{C}$. An aliquot consisting of $10 \%$ of total beads was removed before RNA isolation from each sample for the following immunoprecipitated protein analysis by western blot. After washes, pellets of beads were resuspended in $500 \mu$ of Trizol Reagent (Invitrogen Life Technologies, Paisley, UK) and RNAs were eluted following the manufacturer's instructions. The immunoprecipitated RNAs with FUS and IgG control were quantized $(\mathrm{ng} / \mu \mathrm{l})$ using a NanoDrop 2000 spectrophotometer (Thermo, Waltham, MA, United States) and 
used for circCNOT6L and CNOT6L-mRNA qRT-PCR analysis, using respective primers.

\section{Protein extraction and western blot analysis}

All murine tissues and cells (WT and $\mathrm{CbI}^{-/-}$testes, WT testes in vitro treated \pm ACEA, WT and $\mathrm{CbI}^{-1-}$ epididymal SPZ, total epididymal tissue from WT and $\mathrm{Cbl}^{-1-}$, caput and cauda epididymal tissue from WT, WT caput and cauda SPZ, WT caput SPZ in vitro treated \pm ACEA, human $A$ and B-SPZ fractions and murine E14tg2 $2^{\text {pcDNA3_prZScan4_LNGFR }}$ cells transfected with Fus siRNA or siScr), were separately homogenized in RIPA buffer [PBS, pH 7.4, $10 \mathrm{mM}$ dithiothreitol, $0.02 \%$ sodium azide, $0.1 \%$ SDS, $1 \%$ NP-40, $0.5 \%$ sodium deoxycholate, in the presence of protease inhibitors $(10 \mu \mathrm{g} / \mathrm{ml}$ of leupeptin, aprotinin, pepstatin $\mathrm{A}$, chymostatin, and $5 \mu \mathrm{g} / \mathrm{ml}$ of TPCK)] and sonicated three times for $30 \mathrm{~s}$ bursts, each at $60 \mathrm{~mW}$. Proteins were separated by SDS-PAGE $(8 \%$ acrylamide) and transferred to polyvinylidene difluoride membrane (GE Healthcare, Milano, Italy) at $280 \mathrm{~mA}$ for $2.5 \mathrm{~h}$ at $4{ }^{\circ} \mathrm{C}$. The filters were treated for $3 \mathrm{~h}$ with blocking solution [5\% nonfat milk, $0.25 \%$ Tween-20 in Tris-buffered saline (TBS, $\mathrm{pH}$ 7.6)] and then separately incubated overnight, at $4{ }^{\circ} \mathrm{C}$ in TBS-milk buffer (TBS pH 7.6, 3\% nonfat milk) with different primary antibodies [FUS (PA552610), QKI (PA5-87292), RNApol2 (PA5-86234) from Invitrogen, Milano, Italy, diluted 1:500; ADAR (sc-73408) Santa Cruz Biotechnology, Heidelberg, Germany, diluted 1:500; CB1 C-terminal [32] diluted 1:500; alpha-Tubulin (sc-5286) Santa Cruz Biotechnology, Heidelberg, Germany, diluted 1:1000; MFG-E8 (sc-377356) Santa Cruz Biotechnology, Heidelberg, Germany, diluted 1:1000; Actin (E-AB-20034) Elabscience Biotechnology, Wuhan, China, diluted 1:1000]. After washing in $0.25 \%$ Tween 20 TBS, filters were incubated with 1:1000 horseradish peroxidase-conjugated rabbit IgG (Dako Corp., Milano, Italy) in TBS-milk buffer and then washed again. The immune complexes were detected using the enhanced chemiluminescence-western blotting detection system [Amersham ECL western Blotting Detection Reagent (RPN2106) GE Healthcare, Milano, Italy]. Signals were quantified by densitometry analysis, adjusted relatively to Tubulin (TUB) or Actin (ACT) levels and graphed as fold change (fc) of OD values and reported as mean \pm SEM. The specificity of the immunoreactions was routinely checked by omitting primary $\mathrm{Ab}$ (data not shown).

\section{Protein immunoprecipitation (IP)}

For IP, $1 \times 10^{7}$ of mice caput SPZ or human B-SPZ were lysed with RIPA buffer, in the presence of protease inhibitors
(10 $\mu \mathrm{g} / \mathrm{ml}$ of leupeptin, aprotinin, pepstatin A, chymostatin, and $5 \mu \mathrm{g} / \mathrm{ml}$ of TPCK), sonicated three times for $30 \mathrm{~s}$ bursts, each at $60 \mathrm{~mW}$ and then incubated on ice for $30 \mathrm{~min}$. Lysates were centrifuged at maximum speed for $30 \mathrm{~min}$ $4{ }^{\circ} \mathrm{C}$ and $500 \mu \mathrm{g}$ of supernatant proteins from each sample was incubated with $2 \mu \mathrm{g}$ of relative Ab [FUS (PA5-52610), QKI (PA5-87292), RNApol2 (PA5-86234) from Invitrogen, Milano, Italy] or IgG (12370; Sigma-Aldrich, Milano, Italy) under rotary agitation at $4{ }^{\circ} \mathrm{C}$ overnight. Afterwards, Protein A/G PLUS Agarose Beads (sc-2003; Santa Cruz Biotechnology, Heidelberg, Germany) were added to each sample for $4 \mathrm{~h}$, at $4{ }^{\circ} \mathrm{C}$ under rotary agitation. After bead incubation, samples were washed three times $\left(3000 \times \mathrm{g}\right.$ for $3 \min$ a $\left.4{ }^{\circ} \mathrm{C}\right)$ in $500 \mu$ of cold TBS pH 7.6 and boiled in Laemmli sample buffer for $10 \mathrm{~min}$ to be later analyzed by SDS-PAGE.

\section{Functional Annotation for circRNA/miRNA and Target miRNA Interaction}

Validated or predicted targets of miRNAs were retrieved by Diana TarBase 8.0 (http://www.microrna.gr/tarbase); circRNA/miRNA/Target network was built and visualized using Bisogenet plug-in of Cytoscape (https://cytoscape. org/).

\section{In silico analysis of circCNOT6L}

RBP binding sites matching to circRNAs and flanking regions of circRNAs were predicted through CircInteractome (https://circinteractome.nia.nih.gov/index.html) (26669964). The identification of the internal ribosome entry sites (IRES) and the open reading frame (ORF) for the circRNA with protein coding potential was performed by circRNADb (version1.0.0) (http://202.195.183.4:8000/ circrnadb/circRNADb.php) (27725737). Protein-RNA interaction networks from CLIP-Seq data sets were predicted through starBase v2.0 (http://starbase.sysu.edu.cn/).

\section{Correlation analysis}

All the circCNOT6L and FUS expression values relative to caput and cauda SPZ or epididymis of WT mice have been correlated with each other. Specifically, we included in our analysis both caput and cauda SPZ or epididymal tissue, without considering the epididymal region. Data were compared using the Excel built-in distribution functions available in Microsoft Office. The value of $r$ was considered to establish the test significance. The range $-1 \leq r \leq 1$ established a negative or positive correlation between circCNOT6L and FUS. 


\section{Statistical analysis}

ANOVA followed by Student's $t$-test and Duncan's test (for multi group comparison), were used to identify groups having different mean. Differences with $P<0.05$ were considered statistically significant. Data were expressed as the mean \pm SEM from at least three independent animals for each genotype or experimental group. For qRT-PCR and western blot analyses, triplicates from each of three animals/ genotype or experimental group were considered.

\section{Results}

\section{CB1 involvement in circCNOT6L biogenesis in testis}

CircCNOT6L expression has been previously correlated with sperm quality in humans [3]. Based on this, we decided to evaluate the expression levels of circCNOT6L and its mRNA counterpart (CNOT6L-mRNA) in the testis of WT and $\mathrm{Cbl}^{-/-}$, an animal model for poor sperm quality [19, 22]
(Fig. 1A). Results showed that circCNOT6L levels were significantly lower $(P<0.01)$ in $\mathrm{Cbl}^{-/-}$than in WT testis, while CNOT6L-mRNA levels were significantly higher $(P<0.01)$ in absence of CB1, suggesting that the loss of CB1 affected circ- and CNOT6L-mRNA testicular content (Fig. 1A).

In the same animals, we verified the testicular levels of FUS and ADAR proteins, considering their possible involvement in circRNA biogenesis, by western blot analysis (Fig. 1B and C). The quantitative densitometry analysis of signals showed a significantly lower FUS content $(P<0.01)$ in $\mathrm{Cbl}^{-1-}$ than in WT testis (Fig. 1B); conversely, ADAR levels were higher $(P<0.01)$ in $\mathrm{CbI}^{-/-}$compared to WT testis (Fig. 1C).

The possible participation of CB1 in circCNOT6L biogenesis was then verified by incubating WT testes with vehicle or ACEA-a selective cannabinoid CB1 receptor agonist-at increasing concentrations $(0.1 \mu \mathrm{M}, 1 \mu \mathrm{M}$ and $10 \mu \mathrm{M})$. After the incubation, WT testes were processed to quantify: (i) circCNOT6L (Fig. 1D) and CNOT6L-mRNA levels (Fig. 1E) by qRT-PCR analysis; (ii) FUS (Fig. 1F) and ADAR expression (Fig. 1G) by western blot analysis.
A

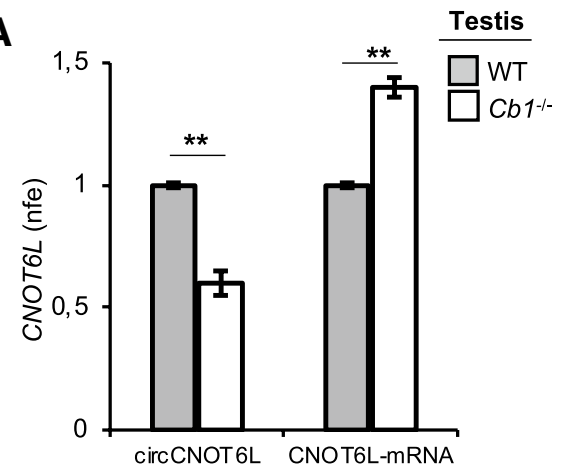

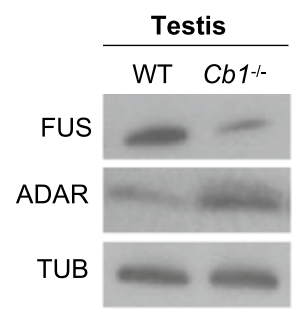

B

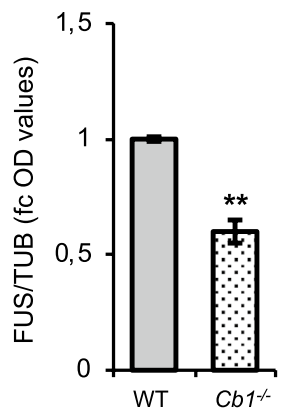

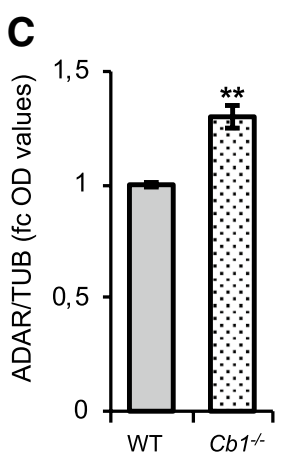

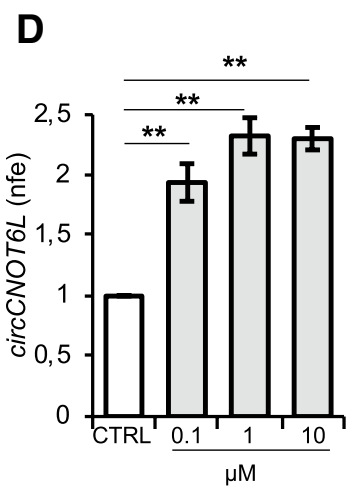

E

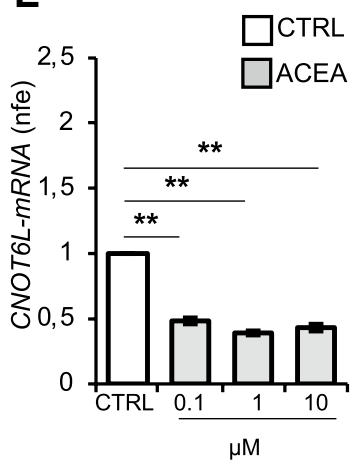

Fig. 1 CB1 involvement in circCNOT6L biogenesis in testis. qRTPCR detection of circCNOT6L and CNOT6L-mRNA expression levels (A); immunoblots and quantification of FUS (B) and ADAR (C) proteins in testis of WT and $\mathrm{Cbl}^{-/}$adult mice $(n=3$ mice in triplicate for each group). qRT-PCR detection of circCNOT6L (D), CNOT6L-mRNA (E) expression levels and immunoblots and quantification of FUS $(\mathbf{F})$ and $\operatorname{ADAR}(\mathbf{G})$ proteins in testis of WT mice

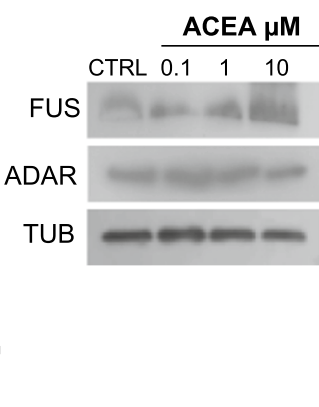

$\mathbf{F}$

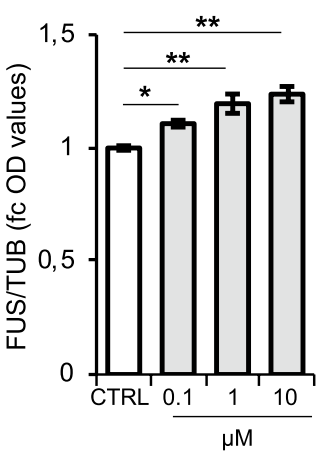

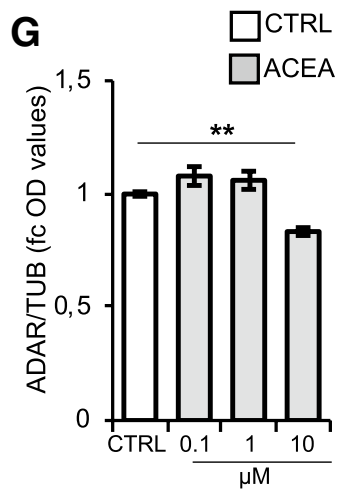

in vitro treated with vehicle (CTRL) or ACEA at different concentrations: $0,1-1-10 \mu \mathrm{M}(n=3$ different testes in triplicate for each experimental group). In (A), (D) and (E), data are reported as mean value of nfe \pm SEM, using Actin as endogenous control, while in $(\mathbf{B}),(\mathbf{C})$, (F) and (G), FUS and ADAR amount was quantified by densitometry analysis, normalized against Tubulin (TUB) signals, expressed as $\mathrm{fc}$ of $\mathrm{OD}$ values and reported as mean value \pm SEM. $* * P<0.01$ 
Results showed that ACEA significantly $(P<0.01)$ increased circCNOT6L levels (Fig. 1D), while a negative effect was observed $(P<0.01)$ on CNOT6L-mRNA levels (Fig. 1E) at all doses in comparison to control group (CTRL).

ACEA treatment significantly increased FUS testicular levels at doses of $0.1 \mu \mathrm{M}, 1 \mu \mathrm{M}(P<0.05)$ and $10 \mu \mathrm{M}$ $(P<0.01)$ in comparison to CTRL group (Fig. $1 \mathrm{~F})$; conversely, ADAR testicular levels significantly decreased at higher dose of $10 \mu \mathrm{M}(P<0.01)$ (Fig. 1G), suggesting that circCNOT6L increase after ACEA treatment may be dependent on the modulation of FUS and ADAR enzymes.

These results clearly suggest that CB1 modulates circCNOT6L biogenesis in testis.

\section{Effects of $\mathrm{Cb} 1$ deletion on circCNOT6L biogenesis in total epididymal SPZ}

With the aim of investigating the possibility that SPZ may be able of a backsplicing activity, total epididymal SPZ from WT and $\mathrm{Cbl}^{-/-}$mice were collected and purified to evaluate: (i) circCNOT6L and CNOT6L-mRNA content by qRT-PCR analysis (Fig. 2A), (ii) FUS (Fig. 2B) and ADAR (Fig. 2C) proteins by western blot analysis, and (iii) sperm FUS localization (Fig. 2D) by immunofluorescence analysis.

$\mathrm{CbI}^{-/-} \mathrm{SPZ}$ had a significant lower and higher content of circCNOT6L and CNOT6L-mRNA, respectively, than WT SPZ ( $<<0.01$, Fig. $2 \mathrm{~A}$ ), thus to reflect what already found in the testis.

The quantitative densitometry analysis of signals showed a significant lower $(P<0.01)$ FUS content in $\mathrm{Cbl}^{-/-}$than in WT SPZ (Fig. 2B); conversely, ADAR levels were higher $(P<0.01)$ in $\mathrm{Cbl}^{-/-}$compared to WT sperm cells (Fig. 2C).

Interestingly, in WT SPZ, FUS localization was clearly defined in peri-acrosomal region and along the entire length of the tail (Fig. 2D). In addition, some WT SPZ showed FUS localization confined to the mid-piece. Conversely, in $\mathrm{Cbl}^{-/-}$SPZ, although FUS localization was similar to WT, the signal strength was lower or completely absent in comparison to WT (Fig. 2D).

To assess a potential epididymal production of key modulators of backsplicing that could be shared with SPZ in transit, SPZ-deprived epididymis pieces from WT and $\mathrm{CbI}^{-/-}$mice were processed to analyze the expression levels of FUS (Fig. 2E) and ADAR (Fig. 2F) by western blot analysis. Results showed a significant decrease of FUS (Fig. 2E; $P<0.01$ ) and increase of ADAR (Fig. $2 \mathrm{~F} ; P<0.01$ ) levels in the epididymal tissue from $\mathrm{CbI}^{-/-}$compared to WT mice.

These results suggest that SPZ contain the molecular modulators for backsplicing; their profile between WT and $\mathrm{Cbl}^{-/-}$well matched with testicular and epididymal ones.

\section{Differential analysis of circCNOT6L in WT caput and cauda SPZ and epididymal tissue}

Considering that along the epididymis, SPZ mature differentially depending on the region (caput or cauda) where they stay, the sperm samples from caput and cauda epididymis of WT mice were isolated and processed to analyze: (i) circCNOT6L and CNOT6L-mRNA expression levels (Fig. 3A) by qRT-PCR analysis; (ii) FUS (Fig. 3B) and ADAR (Fig. 3C) proteins by western blot analysis, and (iii) sperm FUS localization (Fig. 3D and E) by immunofluorescence analysis.

Results showed that circCNOT6L levels significantly increased $(P<0.01)$ in SPZ from caput to cauda epididymis, while CNOT6L-mRNA levels significantly decreased $(P<0.01)$ in cauda $\mathrm{SPZ}$ in comparison to caput $\mathrm{SPZ}$ (Fig. 3A).

FUS and ADAR proteins showed very weak signals: FUS levels were significantly higher $(P<0.05)$ in cauda than in caput SPZ (Fig. 3B); conversely, ADAR levels were lower $(P<0.05)$ in cauda in comparison to caput SPZ (Fig. 3C). FUS localization in caput WT SPZ showed a defined regionalization in peri-acrosomal region and in the mid-piece of sperm tail (Fig. 3D). Differently, in cauda WT SPZ the periacrosomal region appeared positive for FUS signal, but more interestingly, the entire length of sperm tail became positive for FUS staining (Fig. 3E).

Additionally, caput and cauda SPZ-deprived epididymis pieces from WT mice were processed to analyze: (i) the expression levels of circCNOT6L and CNOT6L-mRNA (Fig. 3G) by qRT-PCR analysis; (ii) FUS (Fig. 3H) and ADAR (Fig. 3I) proteins by western blot analysis.

Results showed a significant increase $(P<0.01)$ of circCNOT6L from caput to cauda epididymis (Fig. 3G). Interestingly, CNOT6L-mRNA levels also significantly increased $(P<0.01)$ from caput to cauda epididymis (Fig. 3G).

A significant increase $(P<0.05)$ of FUS levels was observed in cauda in comparison to caput epididymal tissue (Fig. 3H), whereas ADAR levels significantly decreased $(P<0.05)$ in cauda epididymis (Fig. 3I).

We carried out a correlation analysis between circCNOT6L and FUS levels relative to WT caput and cauda SPZ (Fig. 3F) and epididymal tissue (Fig. 3J), regardless of the epididymal region. Correlation analysis showed that circCNOT6L and FUS levels were positively correlated with each other both in SPZ (Fig. 3F; $r=0.905, P<0.001$ ) and in epididymis (Fig. 3J; $r=0.910, P<0.001)$.

These results suggest that SPZ enhance circCNOT6L biogenesis during their maturation along the epididymis. 

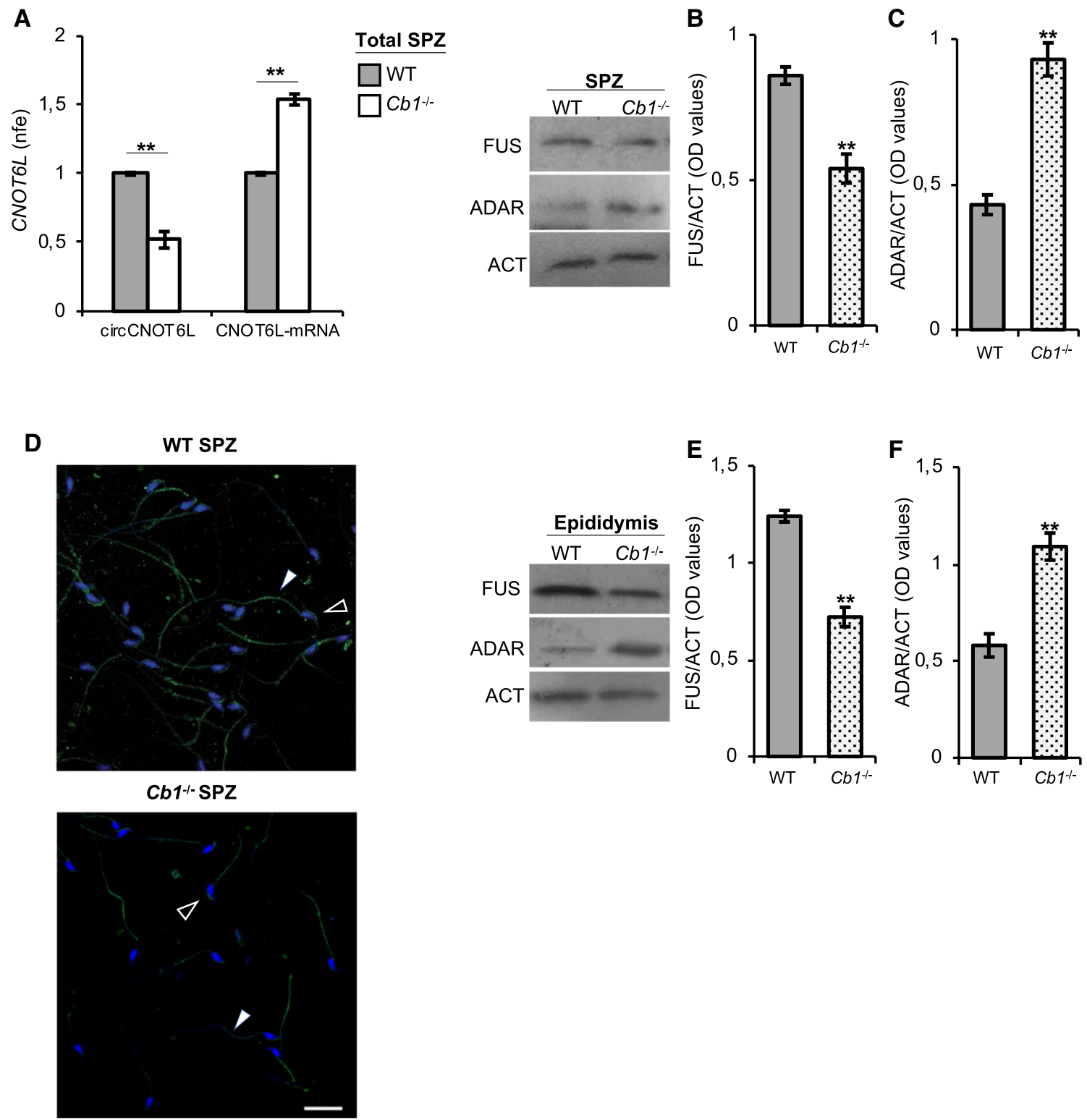

Fig. 2 CB1 involvement in circCNOT6L biogenesis in total epididymal SPZ. qRT-PCR detection of circCNOT6L and CNOT6L-mRNA expression levels (A); immunoblots and quantification of FUS (B and $\mathbf{E})$ and ADAR (C and $\mathbf{F})$ proteins in total epididymal SPZ and/ or epididymal tissue from WT and $\mathrm{Cbl}^{-/-}$adult mice $(n=3$ mice in triplicate for each group). In (A), the data are reported as mean value of nfe \pm SEM, using Actin as endogenous control, while in $(\mathbf{B}),(\mathbf{C})$,
(E) and (F), FUS and ADAR amount was quantified by densitometry analysis, normalized against Actin (ACT) signals, expressed as OD values and reported as mean value \pm SEM. $* * P<0.01$. Immunofluorescence analysis of FUS protein in WT and $\mathrm{Cbl}^{-1-} \mathrm{SPZ}(\mathbf{D})$. White empty arrowheads and white full arrowheads represent FUS localization (FITC-green) in sperm head and tail, respectively. Nuclei were labeled with DAPI (blue). Scale bar: $20 \mu \mathrm{M}$

\section{FUS drives CNOT6L backsplicing by recruiting RNApol2 and QKI in caput SPZ}

To evaluate the physical interaction between FUS and
CNOT6L, both circRNA and mRNA, we carried out a RIP assay in WT caput SPZ, using FUS Ab. The results showed a 15.5- and 8.5-fold enrichment of circCNOT6L and CNOT6L-mRNA, respectively, when the anti-FUS Ab 

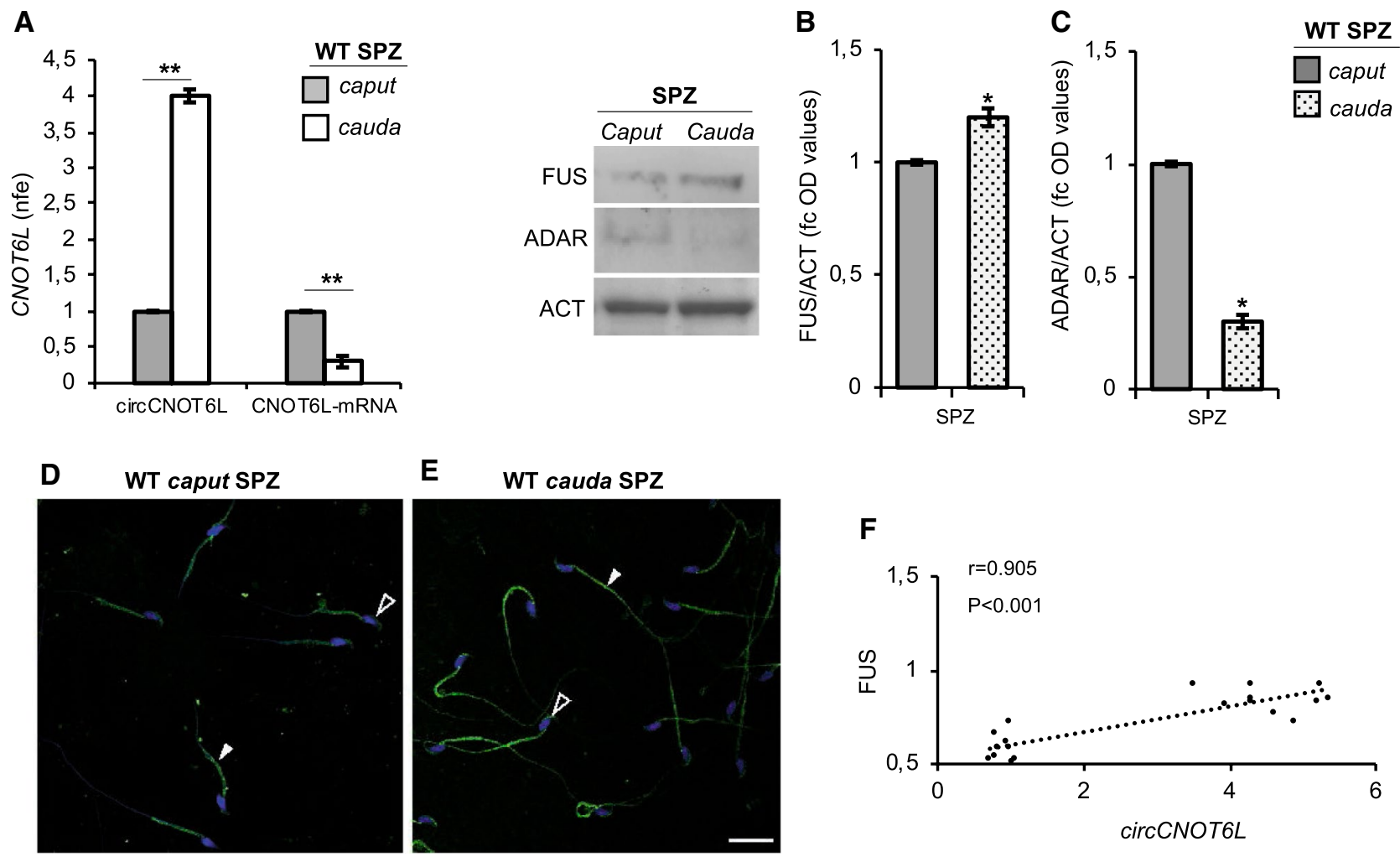

G

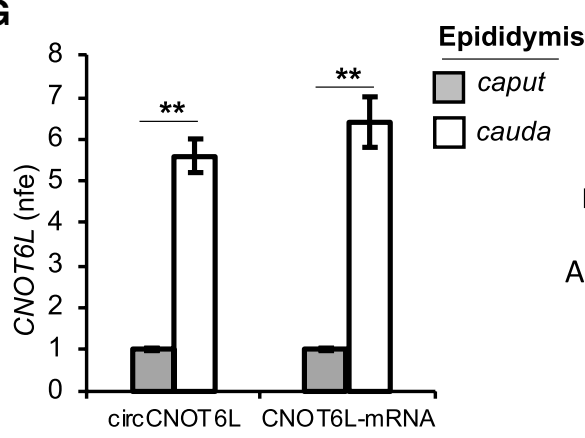

J

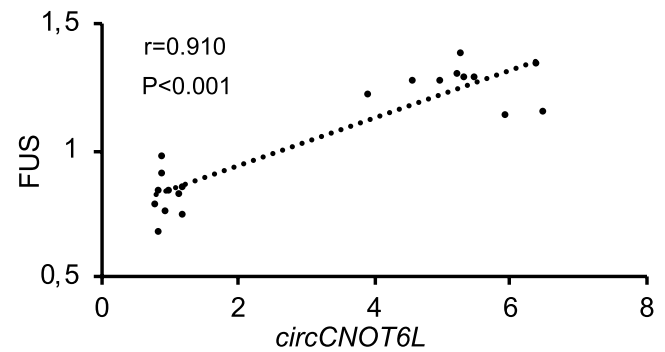

was used, relatively to the use of IgG control (Fig. 4A), reinforcing the idea of a direct interaction of FUS protein with both circCNOT6L and CNOT6L-mRNA.

Accordingly, using starBase v2.0 (http://starbase.sysu. edu.cn/), we identified some putative RBPs able to interact with circCNOT6L, focusing our attention on the ten most
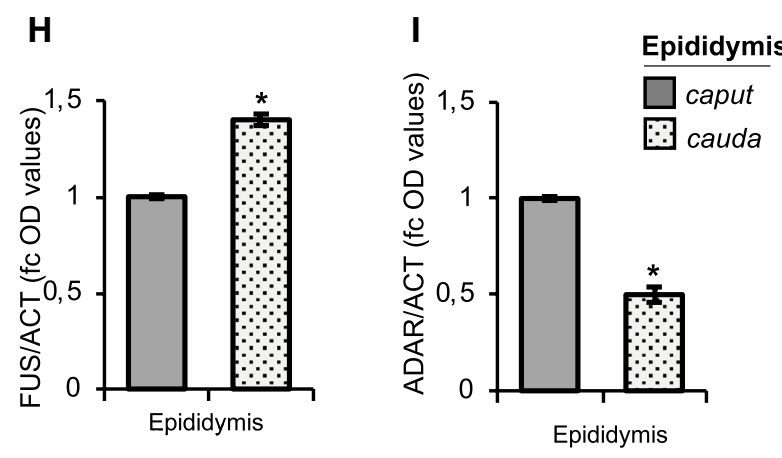

representative (Table 2). Interestingly, the bioinformatic analysis showed that FUS protein possessed a high number of potential target sites for the binding to circCNOT6L (Table 2).

Furthermore, to investigate the possibility that FUS may cooperate with other RBPs to promote circCNOT6L 
4Fig. 3 Differential analysis of circCNOT6L in WT caput and cauda SPZ and epididymal tissue. qRT-PCR detection of circCNOT6L and CNOT6L-mRNA expression levels (A and $\mathbf{G}$ ); immunoblots and quantification of FUS (B and $\mathbf{H})$ and $\operatorname{ADAR}(\mathbf{C}$ and $\mathbf{I})$ proteins in caput and cauda SPZ (A, B and C) and epididymal tissue (G, H and I) from WT adult mice $(n=3$ different samples from three different mice in triplicate). In (A) and $(\mathbf{G})$ data are reported as mean value of nfe \pm SEM, using Actin as endogenous control, while in $(\mathbf{B}),(\mathbf{C})$, (H) and (I), FUS and ADAR amount was quantified by densitometry analysis, normalized against Actin (ACT) signals, expressed as fc of OD values and reported as mean value \pm SEM. $* P<0.05$, $* * P<0.01$. Immunofluorescence analysis of FUS protein in WT caput and cauda SPZ (D and E). White empty arrowheads and white full arrowheads represent FUS localization (FITC-green) in sperm head and tail, respectively. Nuclei were labeled with DAPI (blue). Scale bar: $20 \mu \mathrm{M}$. Correlation analysis (F and $\mathbf{J}$ ) between circCNOT6L and FUS expression values relative to caput and cauda SPZ (F; $r=0.905$, $P<0.001)$ and epididymal tissue $(\mathbf{J} ; r=0.910, P<0.001)$ of WT mice regardless of the epididymal region

biogenesis, we firstly immunoprecipitated FUS from total proteins of WT caput SPZ (IP-FUS), followed by immunoblotting with FUS, RNApol2 and QKI antibodies (Fig. 4B). The results showed stronger FUS, RNApol2 and QKI signals in IP-FUS as compared with a significantly weaker control signals, suggesting a heterotrimeric complex formation among FUS, RNApol2 and QKI (Fig. 4B). Accordingly, the immunoprecipitation of QKI and/or RNApol2 from sperm proteins (IP-QKI and IP- RNApol2, respectively) and the immunoblotting with the same three antibodies confirmed the existence of the heterotrimeric complex in WT caput SPZ (Fig. 4C and D).

With the aim of strengthening the idea that SPZ may be able to circularize CNOT6L-mRNA through CB1 stimulation, SPZ collected from caput epididymis of WT mice were in vitro treated with $1 \mu \mathrm{M}$ ACEA at $37^{\circ} \mathrm{C}$ for $30 \mathrm{~min}$. After the treatment, SPZ were processed to evaluate circCNOT6L and CNOT6L-mRNA levels by qRT-PCR analysis (Fig. 4E). A very significant increase of circCNOT6L expression was observed after CB1 stimulation by ACEA $(P<0.01)$ in comparison to CTRL (vehicle) group; conversely, CNOT6L-mRNA levels significantly decreased $(P<0.01)$ in the ACEA treated group compared to CTRL (Fig. 4E).

Therefore, CNOT6L-mRNA circularization in caput SPZ may be stimulated by CB1 activation and depend on both FUS/CNOT6L-mRNA and FUS-QKI-RNApol2 heterotrimeric interaction. To demonstrate this, we firstly carried out the RIP assay with FUS Ab after the in vitro treatment of caput SPZ with $1 \mu \mathrm{M}$ ACEA.

Relatively to the use of $\operatorname{IgG}$ control, a significant increase (24.5-fold enrichment) of CNOT6L-mRNA immunoprecipitated with FUS was observed after ACEA treatment. Conversely, the results showed a reduction of FUS-circCNOT6L interaction (Fig. 4F). In addition, the western blot analysis on FUS immunoprecipitated proteins relative to RIP fractions in CTRL and ACEA groups evidenced a strong increase of FUS-QKI-RNApol2 heterotrimeric interaction after ACEA treatment (Fig. 4G). This increase was not dependent on the variations of total protein content, as confirmed by the analysis on input samples (total lysates isolated before the immunoprecipitations), suggesting an effective increased recruitment of the heterotrimeric complex on CNOT6LmRNA (Fig. 4G).

Finally, to further prove that FUS regulates circCNOT6L biogenesis, Fus expression was silenced in murine ESC cells cultured in RM medium, using an RNA interference strategy. A specific pool of siRNA for Fus mRNA (siFus) was transfected at different doses $(5 \mathrm{nM} ; 10 \mathrm{nM} ; 25 \mathrm{nM} ; 50 \mathrm{nM})$; cells were then analyzed at different time points following the transfection ( $48 \mathrm{~h}$ and $72 \mathrm{~h}$ ). In both time points, qRT-PCR analysis showed a significant down-regulation of Fus expression levels $(P<0.01 ; P<0.05)$ in comparison to the relative siScr, at all siFus doses (Fig. 4H and I). Interestingly, in both the analyzed time points the most significant reduction of Fus mRNA levels was observed at $25 \mathrm{nM}$ of siFus (Fig. $4 \mathrm{H}$ and I) that was chosen for further validations. Western blot analysis confirmed a significant reduction of FUS content at $48 \mathrm{~h}(P<0.05)$ and $72 \mathrm{~h}(P<0.01)$ after $25 \mathrm{nM}$ of siFus transfection (Fig. 4J). The expression levels of circCNOT6L after $25 \mathrm{nM}$ of siFus transfection were evaluated by qRTPCR analysis. Results showed that circCNOT6L levels were significantly reduced at $48 \mathrm{~h}(P<0.05)$ and $72 \mathrm{~h}(P<0.01)$ after siRNA transfection (Fig. 4K), confirming that FUS is directly involved in circCNOT6L biogenesis.

These results suggest that-under CB1 stimulation-FUS drives circCNOT6L biogenesis in caput SPZ by recruiting QKI and RNApol2 in a heterotrimeric complex. The direct involvement of FUS in backsplicing activity was proven by using an RNA interference strategy.

\section{In vitro experiment of vesicle shuttle: from epididymis to SPZ}

To assess the potential contribution of the epididymal epithelium in delivering molecules to SPZ, and, therefore, the possible sperm uptake of both CNOT6L-mRNA and FUS protein from the ELF, via epididymosomes, caput SPZ were in vitro incubated with caput or cauda ELF, respectively. The samples were then processed to analyze circCNOT6L and CNOT6L-mRNA expression levels by qRT-PCR analysis (Fig. 5A and B) as well as FUS levels by western blot analysis (Fig. 5C).

Results showed that CNOT6L-mRNA levels did not change in Caput ELF group, but significantly increased in caput SPZ co-incubated with cauda ELF (Cauda ELF group) in comparison to CTRL group $(P<0.01$; Fig. 5B). Conversely, circCNOT6L levels did not change in both experimental groups (Fig. 5A). 
A

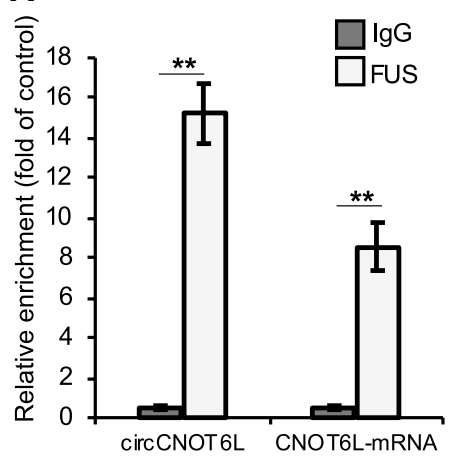

B

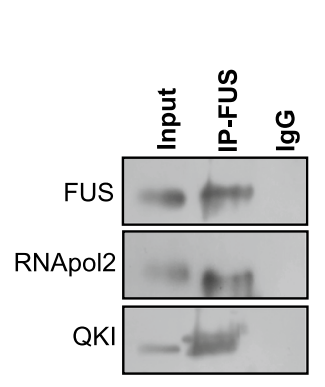

C

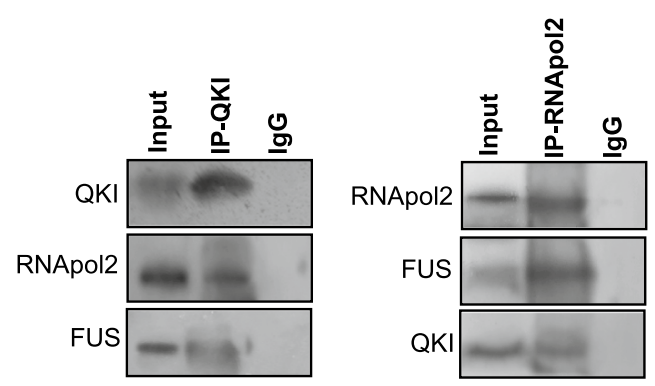

E

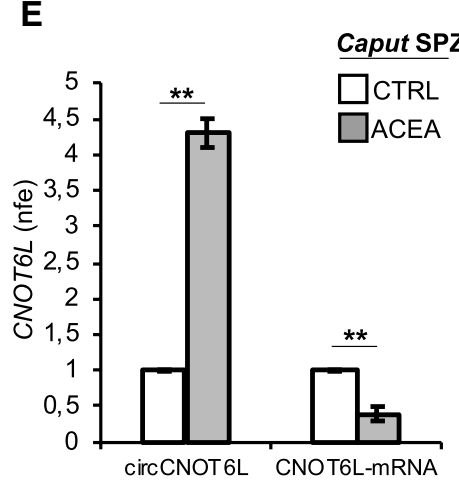

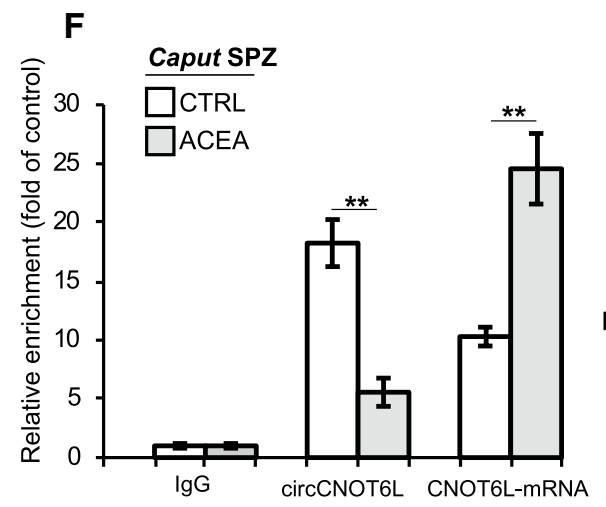
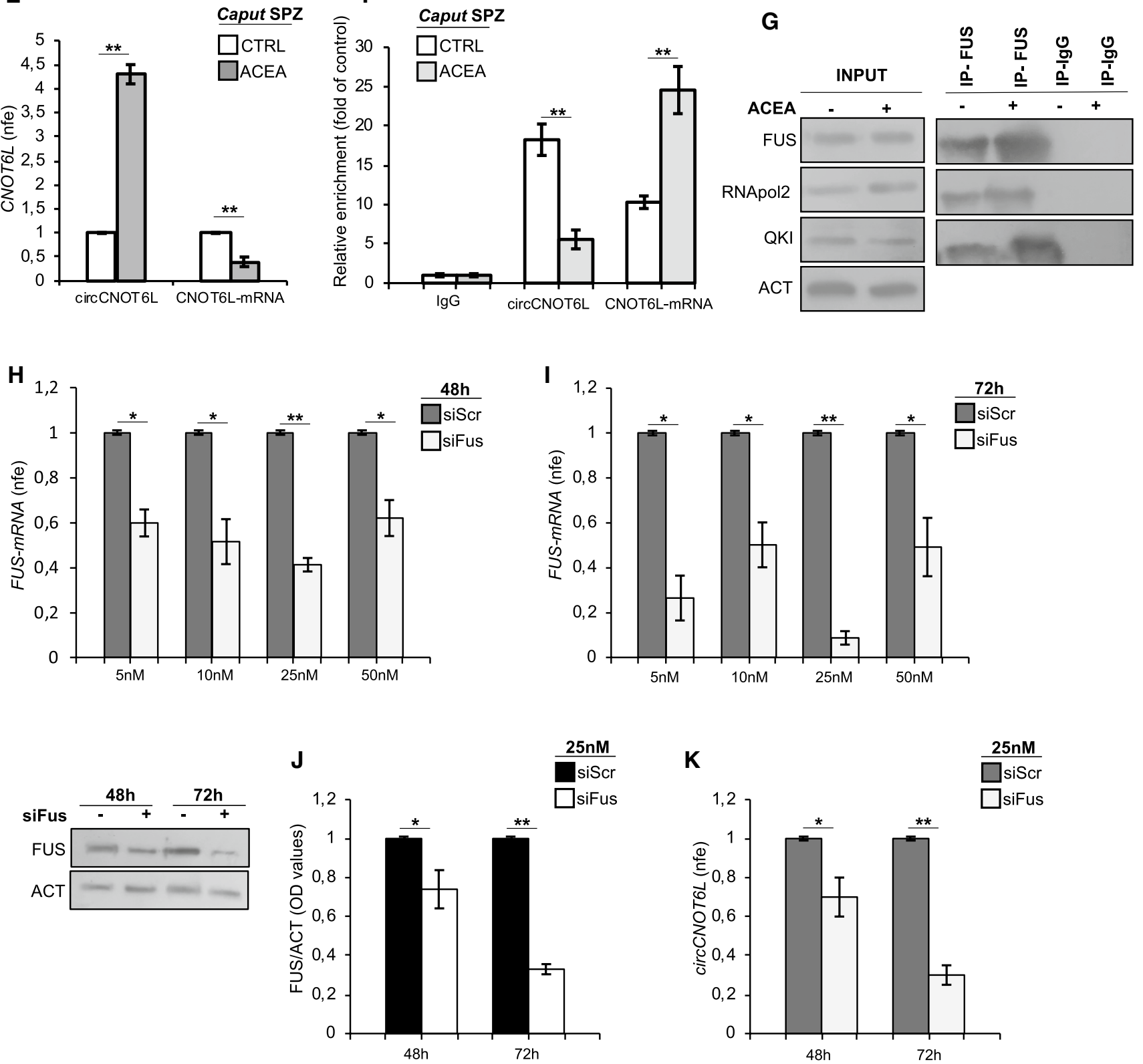
४Fig. 4 FUS drives CNOT6L backsplicing by interacting with RNApol2 and QKI in caput SPZ. The enrichment levels of circCNOT6L and CNOT6L-mRNA in the products of RIP assay (FUS-IP compared with IgG-IP) in WT caput SPZ alone (A) and after in vitro ACEA treatment $(\mathbf{F})$ detected by qRT-PCR. Data are reported as mean \pm SEM from three independent experiments. $* * P<0.01$. qRTPCR detection of circCNOT6L and CNOT6L-mRNA expression levels $(\mathbf{E})$ in caput SPZ from WT mice in vitro treated with vehicle (CTRL) or ACEA $1 \mu \mathrm{M}$ ( $n=3$ different samples from three different animals for each experimental group in triplicate). Data are reported as mean value of $n f e \pm S E M$, using Actin as endogenous control. $* * P<0.01$. Western blot analysis of FUS, QKI and RNApol2 in the products of IP in WT caput SPZ (B, C and D) using FUS, QKI and RNApol2 antibodies. Western blot analysis of RIP protein fraction immunoprecipitated with FUS Ab (FUS-IP) in WT caput SPZ after in vitro ACEA treatment (G). FUS-IP is analyzed in comparison to control IgG-IP and Input protein extracts. qRT-PCR detection of Fus expression levels in ESCs, cultured in RM medium, treated with siScr or siFus $(5,10,25$ and $50 \mathrm{nM})$ and harvested after $48(\mathbf{H})$ and $72 \mathrm{~h}$ (I) from siRNA transfection. Data are reported as mean value of nfe \pm SEM, using Actin as endogenous control. $* * P<0.01 ; * P<0.05$. Western blot analysis of FUS protein (J) and qRT-PCR detection of circCNOT6L expression levels (K) in ESCs, cultured in RM medium, treated with siScr or siFus $(25 \mathrm{nM})$ and harvested after 48 and $72 \mathrm{~h}$ from siRNA transfection. In (J) FUS amount was quantified by densitometry analysis, normalized against Actin (ACT) signals, and reported as mean value of $\mathrm{fc}$ of OD values \pm SEM, while in $(\mathbf{K})$, the data are reported as mean value of nfe \pm SEM, using Actin as endogenous control. $* * P<0.01 ; * P<0.05$

Since the observed CNOT6L-mRNA transfer to SPZexclusively-via cauda ELF, we counteracted such an effect by an anti-CD9 masking Ab approach, previously suggested to reduce the efficacy of protein transfer from epididymosomes to SPZ [26] — prior of co-incubation with caput sperm. Interestingly, CNOT6L-mRNA levels returned to CTRL values (Cauda ELF + CD9 group, $P<0.01$, Fig. 5B). CircCNOT6L levels still remained unchanged (Fig. 5A).

To verify a possible CB1 contribution in circRNA biogenesis, downstream the CNOT6L-mRNA transfer via vesicle shuttle from ELF, caput SPZ were co-incubated with cauda ELF in the presence of $1 \mu \mathrm{M}$ ACEA. This dose was chosen on the basis of the results obtained in testis (Fig. 1). ACEA stimulation significantly decreased CNOT6L-mRNA levels $(P<0.01$; Fig. 5B), increasing circCNOT6L ones (Cauda ELF + ACEA group, $P<0.01$, Fig. 5A).

The same experiment was also analyzed in terms of FUS uptake from ELF by western blot analysis. FUS levels did not change when sperm was co-incubated with caput ELF; instead, it significantly increased after cauda ELF co-incubation in comparison to CTRL group $(P<0.05 ;$ Fig. 5C). FUS levels were similar to CTRL in Cauda ELF + CD9 group, whereas they significantly increased in Cauda ELF + ACEA group in comparison to CTRL group $(P<0.05$; Fig. 5C).

MFG-E8 (i.e., milk fat globule-EGF factor 8) was selected as a typical exosome marker of mouse epididymosomes, with the highest abundance in the caput epididymosomes, useful to provide the efficacy of epididymosome cargo transfer into SPZ, considering its undetectable levels in testis [33]. Accordingly, MGF-E8 levels were significantly higher just in Caput ELF group in comparison to CTRL group $(P<0.05$; Fig. 5D).

These results suggest that CNOT6L-mRNA and FUS protein are shuttled from cauda ELF to SPZ.

\section{CircCNOT6L from SPZ to oocyte and then toward the 2-cell-like state}

Considering that circRNAs are able to harbor several miRNAs [34], the construction of a circRNA-dependent network (ceRNET) is useful to shed light on predicted mRNA targets. Thus, we built a ceRNET for circCNOT6L (Fig. 6A). According to the results of bioinformatic prediction, $5 \mathrm{miR}$ NAs were identified: has-miR-592, has-miR-542-3p, hasmiR-628-5p, has-miR-148b-5p and has-miR-570-5p. Several downstream mRNA targets are preferentially known to be associated with embryo development, as in the case of Foxo3 and Sox 9 mRNAs, respectively [35, 36]. Accordingly, Hox genes have important roles in the development of pharyngeal organs [37], Crim1 maintains retinal vascular stability [38], Smyd2 is induced during cell differentiation [39] and Bmp7-secreted by the ventral centre of Xenopus embryois involved in Spemann's organizer [40].

Based on the FUS increase in WT cauda SPZ and the potential circCNOT6L involvement in embryo development, we decided to investigate a possible paternal transmission of FUS as well as CNOT6L, both circ- and mRNA, to oocytes after fertilization.

The analysis was carried out in murine NF, $\mathrm{F}$ - at the time in which male and female pronuclei were not fused yet - and then in PA to exclude a potential maternal contribution on FUS and CNOT6L, both circ- and mRNA, content (Fig. 6B-D).

FUS levels, both mRNA and protein, were significantly higher $(P<0.01)$ in $\mathrm{F}$ oocytes in comparison to NF and PA (Fig. 6E and F). Interestingly, circCNOT6L levels significantly increased $(P<0.01)$ after fertilization in $\mathrm{F}$ experimental group, while CNOT6L-mRNA levels were reduced $(P<0.01$; Fig. $6 \mathrm{G})$; in addition, in PA experimental group both circCNOT6L and CNOT6L-mRNA levels were comparable to their respective levels detected in NF experimental group (Fig. 6G).

With the aim of investigating the role of circCNOT6L after oocyte fertilization during the first phases of zygote development, we carried out expression analysis of CNOT6L-mRNA and circCNOT6L on RA induced transition from ESCs toward the 2-cell-like state [41, 42]. As recently reported, 2-cell-like molecular phenotype is closely associated to 2 cell preimplantation stage and is marked by Zscan4 expression [43]. Since in the absence of RA, Zscan4 
Table 2 CircCNOT6L and its RBPs interactors

\begin{tabular}{llllll}
\hline$R B P$ & $\begin{array}{l}\text { gene- } \\
\text { Name }\end{array}$ & $\begin{array}{l}\text { gene- } \\
\text { Type }\end{array}$ & $\begin{array}{l}\text { cluster- } \\
\text { Num }\end{array}$ & $\begin{array}{l}\text { clipExp- } \\
\text { Num }\end{array}$ & clipIDnum \\
\hline ELAVL1 & CNOT6L & circRNA & 209 & 7 & 227 \\
FMR1 & CNOT6L & circRNA & 68 & 8 & 100 \\
$F U S$ & CNOT6L & circRNA & 46 & 5 & 48 \\
HNRNPA1 & CNOT6L & circRNA & 31 & 7 & 38 \\
CSTF2T & CNOT6L & circRNA & 29 & 7 & 46 \\
PRPF8 & CNOT6L & circRNA & 26 & 4 & 35 \\
RBM47 & CNOT6L & circRNA & 22 & 1 & 22 \\
U2AF1 & CNOT6L & circRNA & 20 & 4 & 34 \\
$R B M 10$ & CNOT6L & circRNA & 9 & 2 & 9 \\
CKI & CNOT6L & circRNA & 3 & 1 & 3 \\
\hline
\end{tabular}

Top 10 RBPs interacting with circCNOT6L as revealed by computational analysis of the circular sequence
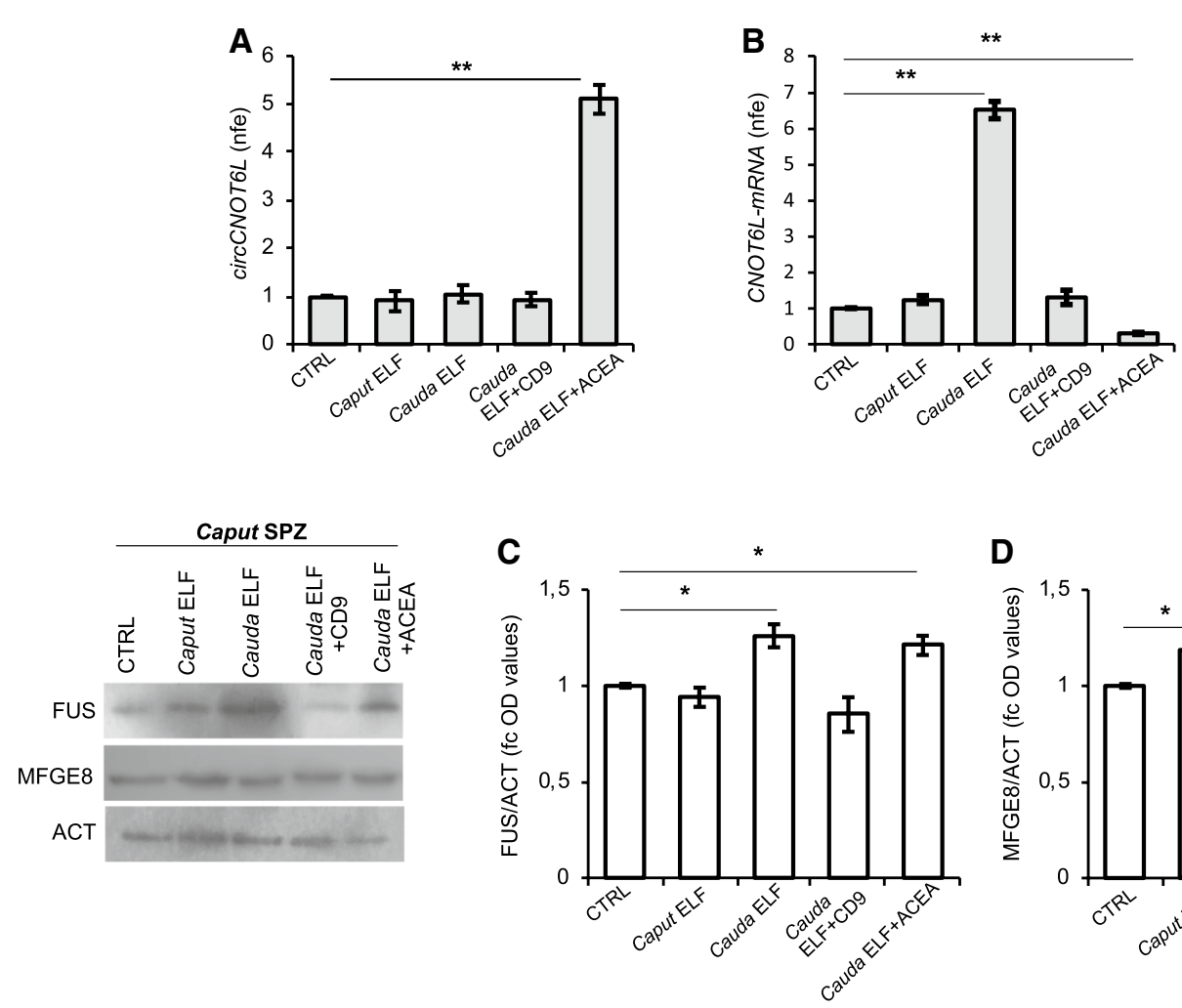

Fig. 5 In vitro experiment of vesicle shuttle: from epididymis to SPZ. qRT-PCR detection of circCNOT6L (A) and CNOT6L-mRNA (B) expression levels in caput SPZ from WT mice in vitro co-incubated with: PBS (CTRL group), Caput ELF, Cauda ELF, Cauda ELF pretreated with anti-CD9 Ab (Cauda ELF + CD9), Cauda ELF in presence of ACEA $1 \mu \mathrm{M}$ (Cauda ELF + ACEA); $(n=3$ different samples for each experimental group from 8 different animals in triplicate). Data are reported as mean value nfe \pm SEM, using Actin as endogenous control. $* * P<0.01$. Immunoblots and quantification of FUS (C)

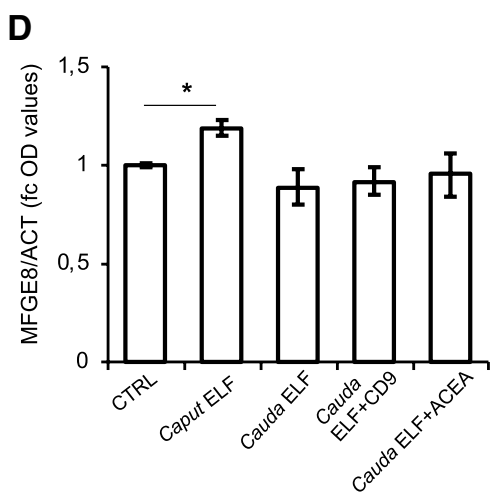

expressing cells (here Zscan $4^{+}$cells) are a small fraction of ESC population (less than 3-5\%), the generation of a modified ESC line harboring Low affinity Nerve Growth Factor Receptor gene (LNGFR) under the control of the Zscan4 promoter, was used as a strategy to efficiently collect homogeneously Zscan $4^{+}$cells [31].

ESCs were cultured in ES RM or ES RM supplemented with RA. Zscan $4^{+}$cells were isolated using a magnetically labeled anti-LNGFR Ab to separate Zscan4 ${ }^{+}$cells $\left(\mathrm{RM}^{+}\right)$ from Zscan4 ${ }^{-}$cells $\left(\mathrm{RM}^{-}\right)$and Zscan $4^{+}$cells $\left(\mathrm{RA}^{+}\right)$from Zscan4- cells $\left(\mathrm{RA}^{-}\right)$.

Expression analysis carried out by qRT-PCR showed a significant reduction of circCNOT6L levels in $\mathrm{RM}^{+}$compared to $\mathrm{RM}^{-}$cells and conversely an increase of CNOT6LmRNA levels in $\mathrm{RM}^{+}$compared to $\mathrm{RM}^{-}$cells (Fig. 6H; $P<0.01)$. Analogously, circCNOT6L levels were lower in $\mathrm{RA}^{+}$than $\mathrm{RA}^{-}$cells, while CNOT6L-mRNA levels were higher in $\mathrm{RA}^{+}$than $\mathrm{RA}^{-}$cells (Fig. $6 \mathrm{I} ; P<0.01$ ).

Finally, comparative analysis between $\mathrm{RM}^{+}$and $\mathrm{RA}^{+}$ cells showed a significant increase of circCNOT6L levels

and MFG-E8 (D) proteins in caput SPZ from WT mice in vitro coincubated with: PBS (CTRL group), Caput ELF, Cauda ELF, Cauda ELF pre-treated with anti-CD9 Ab (Cauda ELF+CD9), Cauda ELF in presence of ACEA $1 \mu \mathrm{M}$ (Cauda ELF + ACEA); $(n=3$ different samples for each experimental group from eight different animals in triplicate). FUS and MFG-E8 amount was quantified by densitometry analysis, normalized against Actin (ACT) signals and expressed as $\mathrm{fc}$ of OD values. Data are reported as mean value of fc of OD values \pm SEM. $* P<0.05$ 
in $\mathrm{RA}^{+}$compared to $\mathrm{RM}^{+}$cells and conversely a reduction of CNOT6L-mRNA levels in $\mathrm{RA}^{+}$compared to $\mathrm{RM}^{+}$cells (Fig. $6 \mathrm{~J} ; P<0.01$ ).

These results suggest the paternal delivery of circCNOT6L to the oocyte. Moreover, the in vitro cell system here analyzed was useful to suggest a possible involvement of circCNOT6L in the zygote transition toward the 2-celllike state.

\section{CircCNOT6L expression and biogenesis in human SPZ}

With the aim of characterizing circCNOT6L expression and biogenesis in human SPZ (h-SPZ), we purified good quality (A-SPZ) and poor quality (B-SPZ) SPZ from normozoospermic volunteers. In detail, circCNOT6L content significantly decreased $(P<0.01)$ in $\mathrm{B}$ - compared to A-SPZ; conversely, CNOT6L-mRNA levels were significantly higher $(P<0.01)$ in B- than in A-SPZ fraction (Fig. 7A).

Additionally, using the same A- and B- sperm populations, we analyzed: (i) CB1, FUS and ADAR protein levels by western blot analysis (Fig. 7B-D), and (ii) sperm FUS localization by immunofluorescence analysis (Fig. 7E and F). CB 1 and FUS levels were significantly higher $(P<0.01)$ in A-SPZ than in B-SPZ (Fig. 7B and C, respectively), while ADAR content was lower $(P<0.01)$ in A-SPZ than in B-SPZ (Fig. 7D).

Immunofluorescence analysis of FUS in A-SPZ showed a clear acrosomal localization and a weak signal in the apical area of the mid-piece of sperm cells (Fig. 7E). In comparison, many B-SPZ were completely negative to FUS immunolocalization although some cells showed the same acrosomal localization of FUS, but with a weaker intensity than A-SPZ (Fig. 7F).

Additionally, the RIP assay in B-SPZ, using FUS Ab, showed a 28.8-fold enrichment of circCNOT6L and 8.2fold enrichment of CNOT6L-mRNA relative to IgG control (Fig. 7G), confirming the direct interaction of FUS protein with both circCNOT6L and CNOT6L-mRNA in B-SPZ.

Furthermore, we immunoprecipitated FUS from total proteins of B-SPZ (IP-FUS), followed by immunoblotting with FUS, RNApol2 and QKI antibodies (Fig. 7H-J). The results showed higher FUS, RNApol2 and QKI levels in IP-FUS as compared to control, suggesting a heterotrimeric complex formation among FUS, RNApol2 and QKI (Fig. 7H). Similarly, the immunoprecipitation of QKI and/or RNApol2 from B-SPZ proteins (IP-QKI and IPRNApol2, respectively) and the immunoblotting with the same three antibodies confirmed the heterotrimeric complex formation in these cells (Fig. 7I and J).

Thus we verified also in humans what already observed in mouse. Using the fraction of B-SPZ isolated from normozoospermic samples, we in vitro treated these with
$1 \mu \mathrm{M}$ ACEA at $37^{\circ} \mathrm{C}$ for $30 \mathrm{~min}$, to evaluate circ- and CNOT6L-mRNA expression (Fig. 7K) by qRT-PCR analysis. CircCNOT6L levels significantly increased in the ACEA treated group $(P<0.01)$ in comparison to CTRL group; conversely, a significant decrease of CNOT6LmRNA levels was observed $(P<0.01)$ (Fig. $7 \mathrm{~K})$.

Finally, to demonstrate CB1 involvement in CNOT6LmRNA circularization in B-SPZ by increasing FUS/ CNOT6L-mRNA interaction, the RIP assay with FUS $\mathrm{Ab}$ was carried out in B-SPZ in vitro treated with $1 \mu \mathrm{M}$ ACEA. As expected, relative to the use of IgG control, a significant 60-fold enrichment of CNOT6L-mRNA immunoprecipitated with FUS was observed after ACEA treatment. Instead, a 7.6-fold enrichment reduction was showed for circCNOT6L (Fig. 7L).

In addition, the western blot analysis on FUS immunoprecipitated proteins relative to RIP fractions in B-SPZ, both CTRL and ACEA-treated, evidenced a strong increase of FUS-QKI-RNApol2 heterotrimeric complex after ACEA treatment (Fig. 7M). This increase was not dependent on the variations of total protein content, as confirmed by the analysis on input samples (total lysates isolated before the immunoprecipitations), confirming an increased recruitment of heterotrimeric complex on CNOT6L-mRNA induced by CB1 activity (Fig. 7M).

These results exactly confirm in h-SPZ the molecular mechanism involved in backsplicing activity, already demonstrated in mouse SPZ.

\section{CircCNOT6L translatability}

To verify the potential translatability of circCNOT6L, we computationally screened the circular sequence to identify interactions with RBPs, ORFs and IRES elements (Table 3). CircCNOT6L may be potentially bound by different RBPs commonly involved in stability, splicing and transport of RNAs, but not in translation. Moreover, circCNOT6L has an ORF from position 11 to $395+5$ generating a protein sequence of 129 amino acids (aa) mostly corresponding to a leucine-rich repeat domain of CNOT6L. Hypothetical IRES elements are localized from positions 357-377 to 244-374; notwithstanding, their R-scores are too low to be considered as bona fide IRES elements.

Accordingly, the possibility that circCNOT6L may be translated is relatively low.

\section{Discussion}

The epigenetic signature of sperm is dynamically responsive to a wide range of environmental and lifestyle stressors [44, 45]. Sperm-derived circRNAs are included 
A

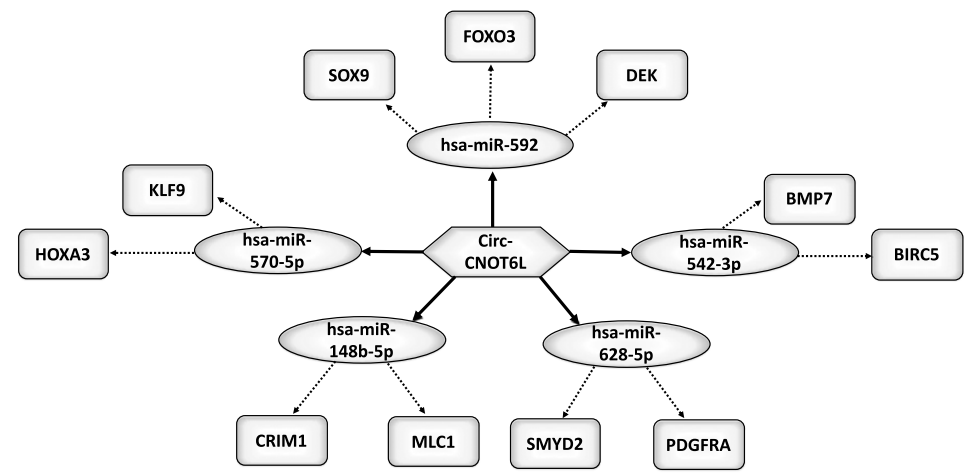

B

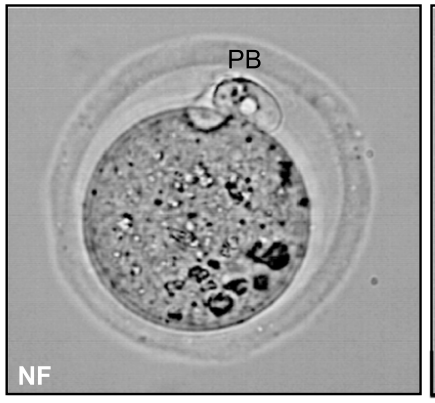

C

D
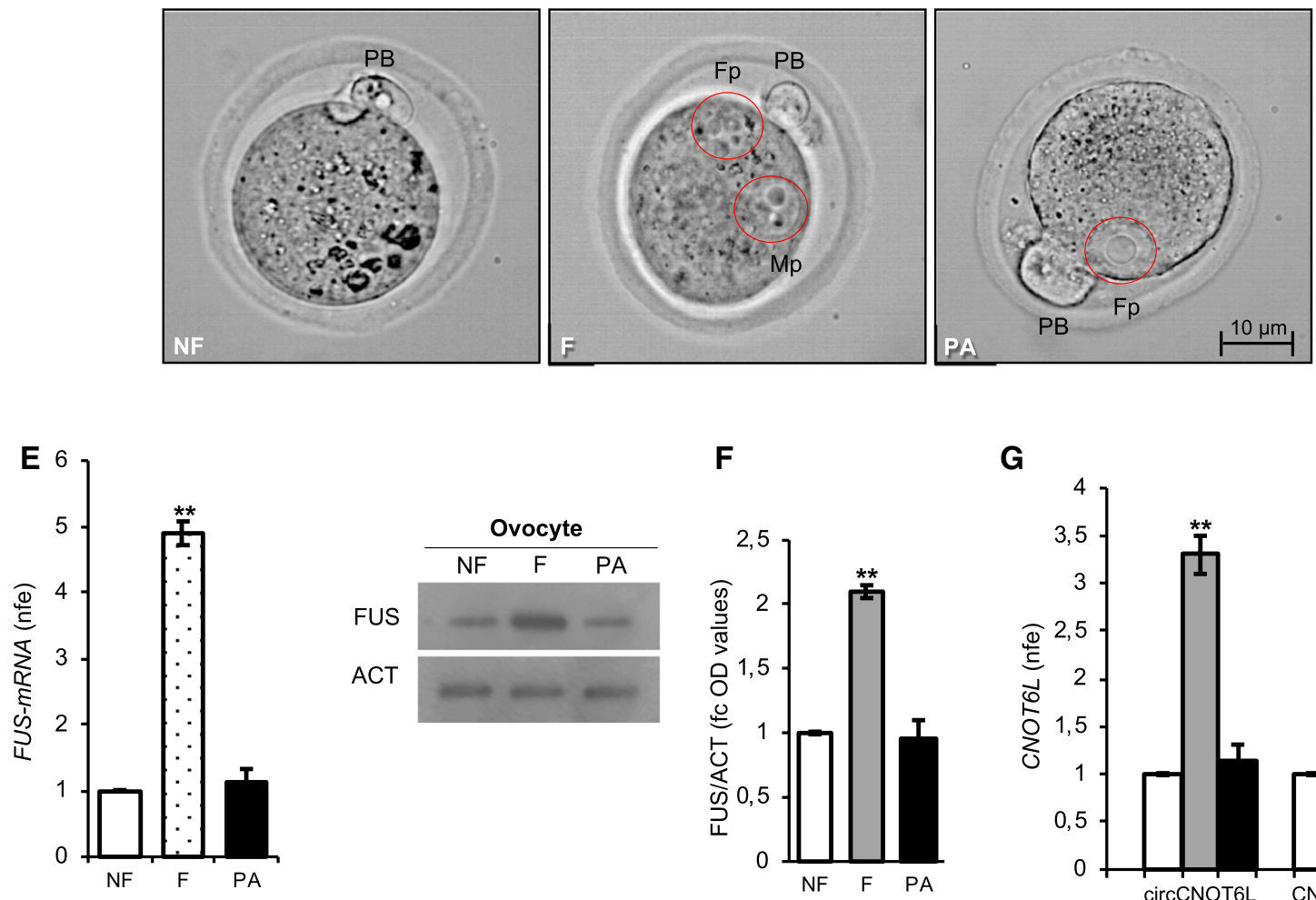

$\mathbf{F}$

G
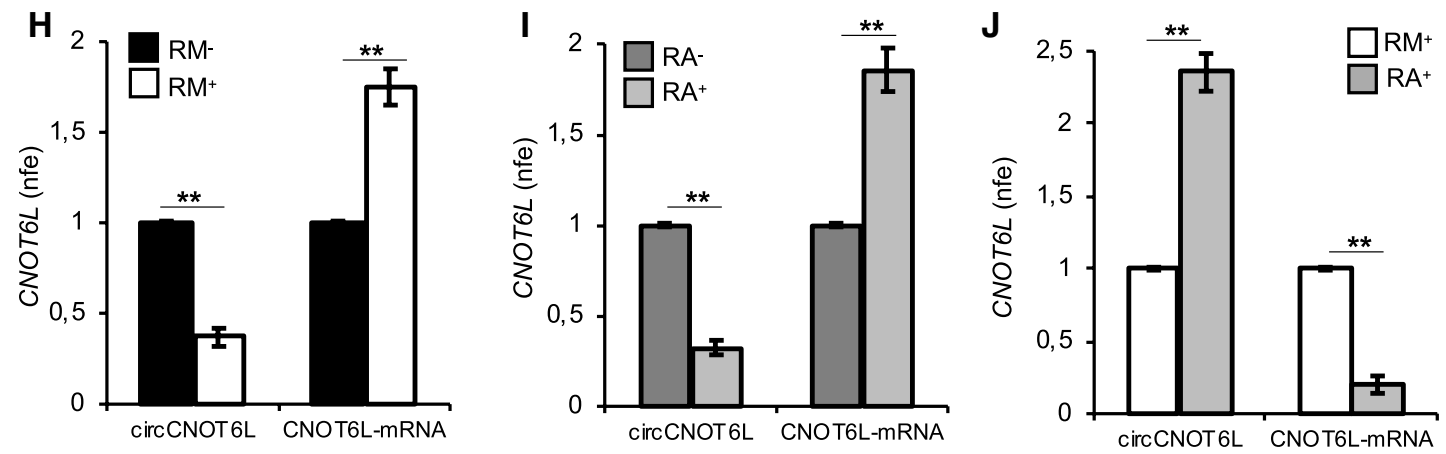

molecules. They have been recently detected in testis, seminal plasma [46-48] and SPZ [3, 27, 49].

Regulators of backsplicing are currently under investigation [50]. Three RBPs are able to bridge intronic sequences in the RNAs: QKI [51], Muscleblind (MBL) [52], and FUS [9]. A functional participation of the RNApol2 in backsplicing has also been suggested; in fact, genes able to produce circRNAs are found to be transcribed at a faster-than-average 
4Fig. 6 circCNOT6L from SPZ to oocyte toward the 2-cell-like state. CircCNOT6L-miRNA-mRNA network analysis was carried out by using the bioinformatic online programs (starBase, circBase, TargetScan, miRBase, Cytoscape) (A). Representative image of NF, F and PA (B, C and $\mathbf{D}) . F p$ female pronucleus, $M p$ male pronucleus, $P B$ first polar globule. Scale bar: $10 \mu \mathrm{M}$. Expression analysis of FUS mRNA $(\mathbf{E})$ and immunoblots and quantification of FUS protein $(\mathbf{F})$ in NF, $\mathrm{F}$ and PA. $n=3$ pools each containing $10 \mathrm{NF}, n=3$ pools each containing $10 \mathrm{~F}$ and $n=3$ pools each containing $10 \mathrm{PA}$ were used for expression analysis. In (E) the data are reported as mean value of nfe \pm SEM using Actin as endogenous control, while in (F), FUS amount was quantified by densitometry analysis, normalized against Actin (ACT) signals, expressed as fc of OD values and reported as mean value \pm SEM. $* * P<0.01$. Expression analysis of circCNOT6L and CNOT6L-mRNA in NF, F, and PA $(\mathbf{G})$ and in ESCs cultured in ES complete medium (RM) or medium supplemented with RA and magnetically separated into $\mathrm{RM}^{-}$(Zscan4 ${ }^{-}$cells), $\mathrm{RM}^{+}$(Zscan4 ${ }^{+}$ cells), $\mathrm{RA}^{-}$(Zscan4 ${ }^{-}$cells) and $\mathrm{RA}^{+}\left(\mathrm{Zscan}^{+}\right.$cells) (H, I and $\mathbf{J}$, respectively) by qRT-PCR. Data are expressed as mean value of nfe \pm SEM, using Actin as endogenous control. $* * P<0.01$

rate from RNApol2 and fruit fly mutants with a lower RNApol2 elongation rate had depleted circRNA levels [52-54]. Instead, degradation pathways and factors, as ADAR, hampering circRNA biogenesis control circRNA turnover [12]. Both FUS and ADAR have been previously analyzed in mammalian testis; the first is preferentially localized in pachytene spermatocytes [55], the second is expressed in both Sertoli and germ cells, mainly spermatogonia [56].

With the aim of investigating circRNA biogenesis in male reproductive tracts, we analyzed the expression of circCNOT6L, already detected in human SPZ of good quality [3], in $\mathrm{Cbl}^{-/-}$male mice because, despite they are fertile, they produce SPZ with poor chromatin quality, DNA fragmentation, and abnormal epididymal motility acquisition [4, 19-23, 57-59]. In $\mathrm{Cbl}^{-/-}$testis and SPZ, circCNOT6L content was negatively affected, as FUS levels, suggesting the possible participation of CB1 in circRNA biogenesis. To assess this hypothesis, we stimulated WT testes with ACEA, a selective cannabinoid CB1 receptor agonist [60], with a dose-response treatment chosen on the basis of pharmacological effectiveness suggested by in vitro experiments carried out in mammals [61, 62]. ACEA induced a significant increase of circCNOT6L to the detriment of its linear counterpart. In this regard, a strong competition exists between canonical splicing and backsplicing considering that both mechanisms share common canonical splice acceptors and donors [63]. However, this aspect is not without challenges due to different experimental approaches/results and therefore needs further investigation. ACEA treatment also influenced the testicular expression of FUS and ADAR proteins that increased and decreased, respectively.

The dormant transcriptional and translational state of sperm cells [64] prompted us to investigate the feasibility of an endogenous backsplicing in SPZ, during their maturation along the epididymis [65-67]. To this scope, circCNOT6L and CNOT6L-mRNA were analyzed in total SPZ collected from WT and $\mathrm{Cbl}^{-/-}$mice, meanwhile FUS and ADAR expression was evaluated in both SPZ and epididymis of WT and $\mathrm{CbI}^{-/-}$mice. All the obtained patterns well matched the testicular profile, reinforcing the idea that CB1 may be involved in the molecular mechanism supporting circCNOT6L biogenesis. Furthermore, FUS localization was assessed in sperm cells by immunofluorescence analysis showing a signal in the peri-acrosomal region and along the tail of WT SPZ; $\mathrm{Cbl}^{-/-} \mathrm{SPZ}$ showed a weaker signal or absence of signal.

The potential contribution of the epididymal epithelium in delivering circRNAs to SPZ was verified by collecting SPZ, separately, from caput and cauda epididymis, as well as caput and cauda epididymis fragments deprived of SPZ. CircCNOT6L and FUS increased in both cauda SPZ and epididymis; ADAR significantly decreased, whereas CNOT6L-mRNA decreased in cauda SPZ and increased in the cauda epididymis. The increased expression of CNOT6L-mRNA in the cauda epididymis may depend on the intense and continuous transcriptional activity of the epididymal epithelial cells. Accordingly, FUS intensity increased in WT SPZ from caput to cauda, extending the positivity from the mid-piece to the entire length of tail. These data would emphasize the support of the epididymal epithelial cells in circRNA biogenesis, darkening a potential ability of sperm cells to do backsplicing. However, if in cauda epididymis SPZ receive the correct amount of circCNOT6L from the epididymal epithelium, how to explain the increase of FUS? And why in cauda SPZ CNOT6L-mRNA significantly decreased? The answer may be that SPZ themselves may have an intrinsic backsplicing ability, supported by an epididymal-dependent uptake of both CNOT6LmRNA and FUS protein.

With this in mind, we designed two experiments.

Firstly, we stimulated WT caput SPZ-producing low levels of circCNOT6L-with ACEA, and observed: (i) a significant increase of circCNOT6L; (ii) a preferential physical interaction between FUS and CNOT6L-mRNA by RIP assay, a powerful method to study the physical association between individual proteins and RNAs, in vivo [68]; (iii) a FUS-mediated recruitment of QKI and RNApol2. In this regard, several circCNOT6L-interacting RBPs were identified by a bioinformatic analysis: FUS protein possessed a high number of potential binding sites; QKI was also included, but at a lesser extent. Instead, RNApol2 was completely absent. However, considering (i) the validated role of QKI in circRNA biogenesis [50], (ii) the functional participation of the RNApol2 in controlling the balance between transcription and canonical splicing that is an important precondition for circRNA production [54], (iii) FUS ability to bind to the C-terminal domain of RNApol2 [69], we aimed 

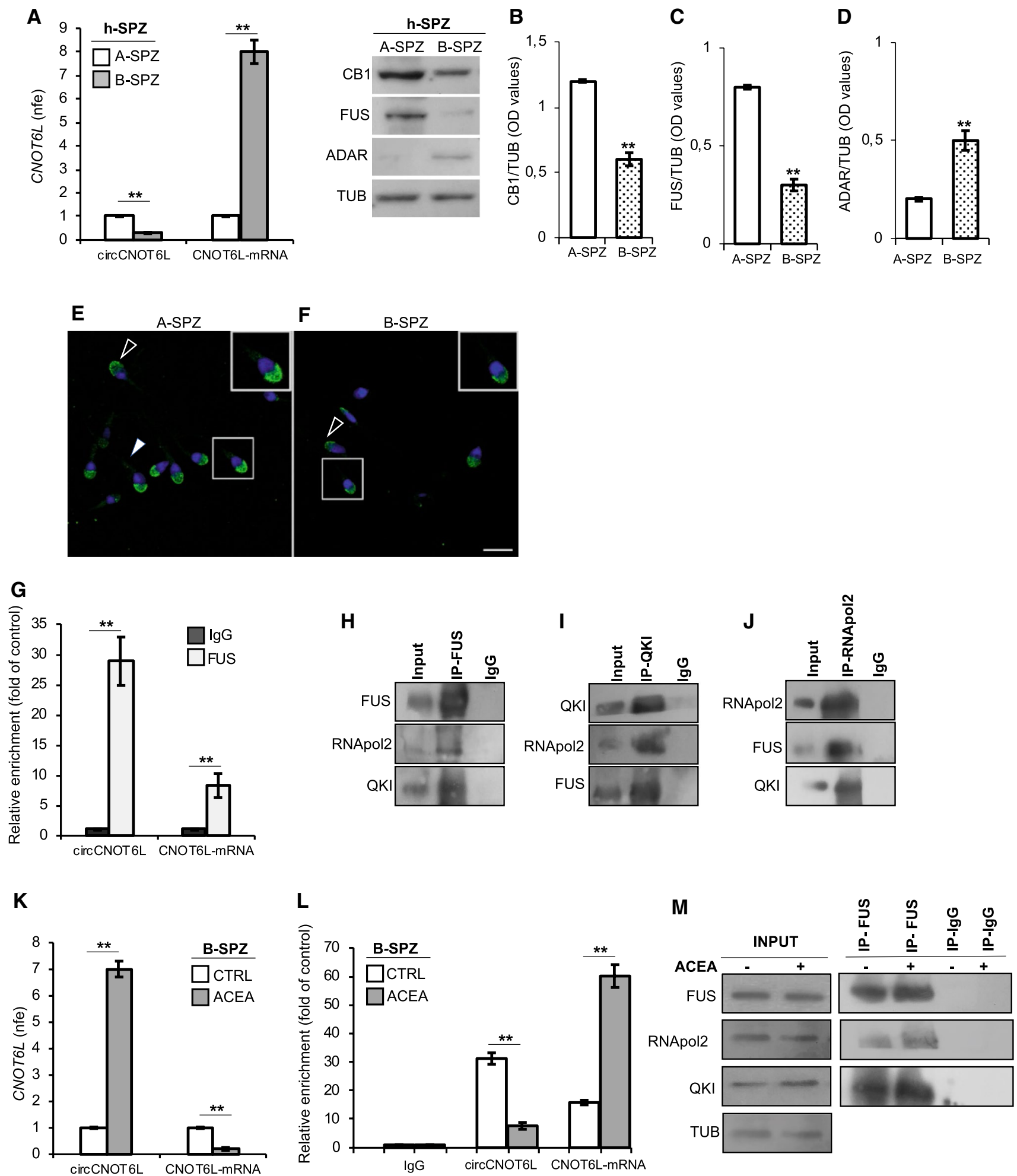

to demonstrate the formation of a heterotrimeric complex among FUS, RNApol2 and QKI in SPZ.

Secondly, WT caput SPZ were in vitro co-incubated with caput or cauda ELF, the fluid known to vehicle epididymosomes $[67,70,71]$. The results suggested that:

(i) CNOT6L-mRNA and FUS protein were shuttled from cauda ELF; (ii) anti-CD9 masking strategy [26, 64, 72, 73] reverted both CNOT6L-mRNA and FUS levels suggesting the epididymosome mediation; (iii) the co-incubation of caput SPZ with ACEA and cauda ELF increased circCNOT6L and decreased CNOT6L-mRNA, confirming that CB1 was a powerful signal for backsplicing reaction. 
४Fig. 7 Human SPZ mimic mouse SPZ. qRT-PCR detection of circCNOT6L and CNOT6L-mRNA expression levels (A); immunoblots and quantification of CB1 (B), FUS (C) and ADAR (D) proteins in A- and B-SPZ fractions from normozoospermic volunteers $(n=5$ different samples in triplicate). Immunofluorescence analysis of FUS protein in A- and B-SPZ (E and $\mathbf{F})$. White empty arrowheads and white full arrowheads represent FUS localization (FITC-green) in sperm head and tail, respectively. Nuclei were labeled with DAPI (blue). Scale bar: $20 \mu \mathrm{M}$. qRT-PCR detection of circCNOT6L and CNOT6L-mRNA expression levels $(\mathbf{G})$ in B-SPZ fractions from normozoospermic volunteers $(n=5)$ in vitro treated with vehicle (CTRL) or ACEA $1 \mu \mathrm{M}(n=5$ different samples for each experimental group in triplicate). In (A) and (G), the data are reported as mean value of nfe \pm SEM, using Gapdh as endogenous control. In (B), (C) and (D) CB1, FUS and ADAR amount was quantified by densitometry analysis, normalized against Tubulin (TUB) signals, expressed as fc of OD values and reported as mean value \pm SEM. $* * P<0.01$. The enrichment levels of circCNOT6L and CNOT6L-mRNA in the products of RIP assay (FUS-IP compared with IgG-IP) in B-SPZ alone $(\mathbf{H})$ and after in vitro ACEA treatment $(\mathbf{L})$ by qRT-PCR. Data are reported as mean \pm SEM from three independent experiments. $* * P<0.01$. Western blot analysis of FUS, QKI and RNApol2 in the products of IP in B-SPZ (I, J and K) using FUS, QKI and RNApol2 antibodies. Western blot analysis of RIP protein fraction immunoprecipitated with FUS Ab (FUS-IP) in B-SPZ after in vitro ACEA treatment (M). FUS-IP is analyzed in comparison to control IgG-IP and Input protein extracts

What may be the functional value of a backsplicing in sperm cells if not to provide a considerable wealth of circRNAs to the oocyte to support embryo development, as the computational analysis was suggesting? Paternal transmission of FUS as well as CNOT6L, both circ- and mRNA, to oocytes, was verified by analyzing murine NF and F, these last chosen at the time in which male and female pronuclei were not fused yet. Although FUS was already identified in murine oocytes by a proteomic approach [74], we observed its increased expression at both mRNA and protein level after fertilization likely to guarantee successful fertilization. To exclude that FUS and circCNOT6L increase in $\mathrm{F}$ may be just a consequence of a maternal activation, we examined PA experimental group. Parthenogenesis-a type of asexual reproduction in which the development of the female gamete takes place without fertilization-is in fact the best strategy to rule out the paternal imprint in the detected backsplicing activity. Interestingly, the content of FUS and CNOT6L, both circ- and mRNA, in PA was similar to NF, strongly supporting our hypothesis of a paternal delivery of molecules into oocyte.

The fate of circCNOT6L during the early phases of zygote development was then explored by analyzing ESC cells toward the 2-cell-like state [41, 42]. RA has been found to induce the zygotic genome activation [43], accompanied by the transition of ESCs to 2-cell-like state, a high pluripotency state [75] and by an upsurge of Zscan $4^{+}$cell population [43]. Interestingly, the functional combined analysis of CNOT6L-mRNA and circCNOT6L in $\mathrm{RM}^{+}$and $\mathrm{RA}^{+}$ cells strongly suggested that circCNOT6L may be an active modulator in zygote transition toward the 2-cell-like state.

What demonstrated in mouse SPZ was then confirmed in humans. SPZ of good quality displayed a high circCNOT6L content, a huge amount of CB1 and significant levels of FUS protein. This firing line suggested that the molecular machinery for backsplicing needs to be switched on to make SPZ suitable for fertilization. In A-SPZ, FUS was localized around the acrosome and in the apical area of the mid-piece; as expected, several B-SPZ were completely negative for FUS immunolocalization. Thus, B-SPZ-containing low levels of circCNOT6L, similarly to mouse caput SPZ-were treated with ACEA to induce backsplicing. CB1 stimulation increased circCNOT6L, by triggering FUS interaction with CNOT6L-mRNA and the formation of FUS-QKI-RNApol2 heterotrimeric complex, as just demonstrated in mouse.

Lastly, although it has been shown that circRNAs can be in vitro and in vivo translated to generate different protein isoforms with specific functional features [4, 76-78], we exclude for circCNOT6L this possibility, according to our computational analysis [77].

In conclusion, as summarized in Fig. 8, our data suggest that along the epididymis, SPZ — not only passively receive a cargo of molecules from the epididymal epithelium - but, even in the absence of de novo gene transcription or protein translation, they are able to circularize linear transcripts through a backsplicing activity and that CB1 may be an important powerful signal.

In addition, our results support the exciting vision of the paternal delivery of molecules intended for embryo development and open new horizons for reproductive biology studies focused on the powerful role of circRNAs in this paternal inheritance of characters.

Acknowledgements We are grateful to Dr. Bruno Ferraro and Dr. Carolina Sellitto (UOSD di Fisiopatologia della Riproduzione, Presidio Ospedaliero di Marcianise, Caserta, Italy) for their technical support in human sperm collection and to Dr. Carmela Belardo (Dipartimento di Medicina Sperimentale, Università degli Studi della Campania "L. Vanvitelli") for her helpful assistance in animal care.

Author contributions Conceptualization: CR, CT, CG and MF; methodology: CR, RM, CD, DA, BD, RL, AM, MF and CT; formal analysis and investigation: $\mathrm{CT}, \mathrm{FG}, \mathrm{AC}, \mathrm{RM}$ and MF; writing-original draft preparation: $\mathrm{CT}, \mathrm{MF}$ and $\mathrm{CR}$; figure preparation: $\mathrm{CT}, \mathrm{RM}, \mathrm{FG}, \mathrm{CD}$, RL and MF; writing - review and editing: CR, PR and CT; visualization: BL, PM and CG; supervision: $\mathrm{CR}$ and $\mathrm{PR}$; funding acquisition: $\mathrm{PR}, \mathrm{CG}$ and CR. 
Table 3 CircCNOT6L and its interactors

\begin{tabular}{lllllll}
\hline RBP & Binding sites & IRES (start-end) & $R$ Score & $\begin{array}{l}\text { Pseu- } \\
\text { doknot } \\
(\mathrm{Y} / \mathrm{N})\end{array}$ & ORF (start-end) & Protein length \\
\hline AGO2 & 2 & $357-377$ & 1.35 & $\mathrm{Y}$ & $11-1$ round +5 nt & 129 aa \\
EIF4A3 & 5 & $244-374$ & 1.33 & $\mathrm{~N}$ & & \\
FMRP & 2 & & & & & \\
IGF2BP2 & 1 & & & & & \\
IGF2BP3 & 2 & & & & & \\
PTB & 1 & & & & & \\
\hline
\end{tabular}

A computational screening of the circular sequence to identify interaction with RBPs, ORFs and IRES elements
Fig. 8 The proposed molecular mechanism at a glance. During the epididymal transit, the activation of $\mathrm{CB} 1$ receptor in SPZ favours the recruitment of the heterotrimeric FUSQKI-RNApol2 complex on CNOT6L-mRNA, thus inducing the backsplicing useful for circCNOT6L biogenesis. In cauda epididymis, a cargo of FUS protein and CNOT6LmRNA is transferred to SPZ via epididymosomes to support the intrinsic ability of SPZ to do backsplicing. After fertilization, the paternal content of FUS, CNOT6L-mRNA and circCNOT6L is transferred from SPZ to oocyte to sustain zygote development

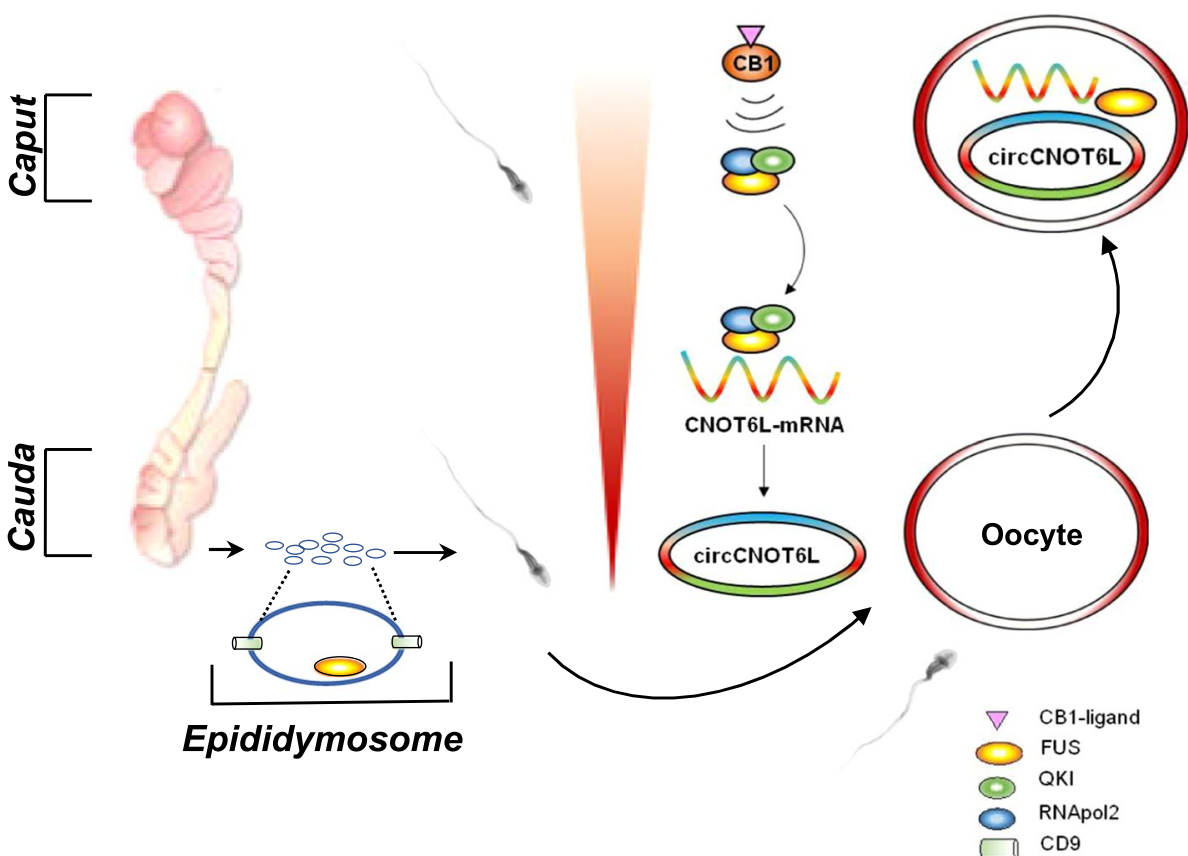

Funding This work was supported by Italian Ministry of University and Research (Grant PRIN to R. Pierantoni 2017); Università degli Studi della Campania (Grant VALERE, Vanvitelli per la Ricerca 2019 to G. Cobellis); Università degli Studi della Campania Luigi Vanvitelli (Grant Dip. Medicina Sperimentale-2020 to R. Chianese).

Availability of data and material The datasets in this study are available from the corresponding author upon reasonable request.

Code availability Not applicable.

\section{Declarations}

Conflict of interest The authors declare that they have no competing interests.

Ethics approval Experiments involving animals were approved by the Italian Ministry of Education and the Italian Ministry of Health, with authorization $\mathrm{n}^{\circ} 941 / 2016$-PR issued on 10.10 .2016 . Procedure involving animal care were carried out in accordance with the National Research Council's publication Guide for Care and Use of Labora- tory Animals (National of Institutes of Health Guide). All procedures involving human participants were in accordance with the 1964 Helsinki Declaration and were carried with the principles of WHO guidelines. This study was approved by Regione Campania-acting as Azienda Sanitaria Locale (ASL) Caserta-prior agreement of ethics committee (n. 1353 del 27.10.2017).

Consent to participate Written informed consent was obtained from all individual participants included in the study.

Consent for publication Consent for publication is not applicable to this study.

Open Access This article is licensed under a Creative Commons Attribution 4.0 International License, which permits use, sharing, adaptation, distribution and reproduction in any medium or format, as long as you give appropriate credit to the original author(s) and the source, provide a link to the Creative Commons licence, and indicate if changes were made. The images or other third party material in this article are included in the article's Creative Commons licence, unless indicated otherwise in a credit line to the material. If material is not included in 
the article's Creative Commons licence and your intended use is not permitted by statutory regulation or exceeds the permitted use, you will need to obtain permission directly from the copyright holder. To view a copy of this licence, visit http://creativecommons.org/licenses/by/4.0/.

\section{References}

1. Salzman J, Gawad C, Wang PL, Lacayo N, Brown PO (2012) Circular RNAs are the predominant transcript isoforms from hundreds of human genes in diverse cell types. PLoS One 7:e30733. https://doi.org/10.1371/journal.pone.0030733

2. Memczak S, Jens M, Elefsinioti A, Torti F, Krueger J, Rybak A, Maier L, Mackowiak SD, Gregersen LH, Munschauer M et al (2013) Circular RNAs are a large class of animal RNAs with regulatory potency. Nature 495:333-338. https://doi.org/10.1038/ nature 11928

3. Chioccarelli T, Manfrevola F, Ferraro B, Sellitto C, Cobellis G, Migliaccio M, Fasano S, Pierantoni R, Chianese R (2019) Expression patterns of circular RNAs in high quality and poor quality human spermatozoa. Front Endocrinol (Lausanne) 10:435. https:// doi.org/10.3389/fendo.2019.00435

4. Chioccarelli T, Pierantoni R, Manfrevola F, Porreca V, Fasano S, Chianese R, Cobellis G (2020) Histone post-translational modifications and CircRNAs in mouse and human spermatozoa: potential epigenetic marks to assess human sperm quality. J Clin Med 9:E640. https://doi.org/10.3390/jcm9030640

5. Chen LL, Yang L (2015) Regulation of circRNA biogenesis. RNA Biol 12:381-388. https://doi.org/10.1080/15476286.2015.10202 71

6. Jeck WR, Sorrentino JA, Wang K, Slevin MK, Burd CE, Liu J, Marzluff WF, Sharpless NE (2013) Circular RNAs are abundant, conserved, and associated with ALU repeats. RNA 19:141-157. https://doi.org/10.1261/rna.035667.112

7. Yu CY, Kuo HC (2019) The emerging roles and functions of circular RNAs and their generation. J Biomed Sci 26:29. https://doi. org/10.1186/s12929-019-0523-Z

8. Guria A, Sharma P, Natesan S, Pandi G (2020) Circular RNAsthe road less traveled. Front Mol Biosci 6:146. https://doi.org/10. 3389/fmolb.2019.00146

9. Errichelli L, Modigliani SD, Laneve P, Colantoni A, Legnini I, Capauto D, Rosa A, De Santis R, Scarfò R, Peruzzi G et al (2017) FUS affects circular RNA expression in murine embryonic stem cell-derived motor neurons. Nat Commun 8:14741. https://doi. org/10.1038/ncomms14741

10. Zhang M, Wang T, Xiao G, Xie Y (2020) Large-scale profiling of RBP-circRNA interactions from public CLIP-Seq datasets. Genes (Basel) 11:54. https://doi.org/10.3390/genes11010054

11. Zang J, Lu D, Xu A (2020) The interaction of circRNAs and RNA binding proteins: an important part of circRNA maintenance and function. J Neurosci Res 98:87-97. https://doi.org/10.1002/jnr. 24356

12. Ivanov A, Memczak S, Wyler E, Torti F, Porath H, Orejuela MR, Piechotta M, Levanon EY, Landthaler M, Dieterich C et al (2015) Analysis of intron sequences reveals hallmarks of circular RNA biogenesis in animals. Cell Rep 10:170-177. https://doi.org/10. 1016/j.celrep.2014.12.019

13. Tang C, Xie Y, Yu T, Liu N, Wang Z, Woolsey RJ, Tang Y, Zhang X, Qin W, Zhang Y (2020) m6A-dependent biogenesis of circular RNAs in male germ cells. Cell Res 30:211-228. https://doi.org/ 10.1038/s41422-020-0279-8

14. Goldstrohm AC, Wickens M (2008) Multifunctional deadenylase complexes diversify mRNA control. Nat Rev Mol Cell Biol 9:337-344. https://doi.org/10.1038/nrm2370
15. Houseley J, Tollervey D (2009) The many pathways of RNA degradation. Cell 136:763-776. https://doi.org/10.1016/j.cell.2009. 01.019

16. Sha QQ, Yu JL, Guo JX, Dai XX, Jiang JC, Zhang YL, Yu C, Ji SY, Jiang Y, Zhang SY et al (2018) CNOT6L couples the selective degradation of maternal transcripts to meiotic cell cycle progression in mouse oocyte. EMBO J 37:e99333. https://doi.org/10. 15252/embj.201899333

17. Horvat F, Fulka H, Jankele R, Malik R, Jun M, Solcova K, Sedlacek R, Vlahovicek K, Schultz RM, Svoboda P (2018) Role of Cnot61 in maternal mRNA turnover. Life Sci Alliance 1:e201800084. https://doi.org/10.26508/1sa.201800084

18. Ricci G, Cacciola G, Altucci L, Meccariello R, Pierantoni R, Fasano S, Cobellis G (2007) Endocannabinoid control of sperm motility: the role of epididymus. Gen Comp Endocrinol 153:320 322. https://doi.org/10.1016/j.ygcen.2007.02.003

19. Chioccarelli T, Cacciola G, Altucci L, Lewis SE, Simon L, Ricci G, Ledent C, Meccariello R, Fasano S, Pierantoni R, Cobellis G (2010) Cannabinoid receptor 1 influences chromatin remodeling in mouse spermatids by affecting content of transition protein 2 mRNA and histone displacement. Endocrinology 151:5017-5029. https://doi.org/10.1210/en.2010-0133

20. Chioccarelli T, Manfrevola F, Porreca V, Fasano S, Altucci L, Pierantoni R, Cobellis G (2020) The cannabinoid receptor CB1 stabilizes sperm chromatin condensation status during epididymal transit by promoting disulphide bond formation. Int J Mol Sci 21:3117. https://doi.org/10.3390/ijms21093117

21. Cobellis G, Ricci G, Cacciola G, Orlando P, Petrosino S, Cascio MG, Bisogno T, De Petrocellis L, Chioccarelli T, Altucci L, Fasano S, Meccariello R, Pierantoni R, Ledent C, Di Marzo V (2010) A gradient of 2-arachidonoylglycerol regulates mouse epididymal sperm cell start-up. Biol Reprod 82:451-458. https://doi.org/10. 1095/biolreprod.109.079210

22. Cacciola G, Chioccarelli T, Altucci L, Ledent C, Mason JI, Fasano S, Pierantoni R, Cobellis G (2013) Low $17 \beta$-estradiol levels in Cnr1 knock-out mice affect spermatid chromatin remodeling by interfering with chromatin reorganization. Biol Reprod 88:1-12. https://doi.org/10.1095/biolreprod.112.105726

23. Cacciola G, Chioccarelli T, Fasano S, Pierantoni R, Cobellis G (2013) Estrogens and spermiogenesis: new insights from type 1 cannabinoid receptor knockout mice. Int J Endocrinol. https://doi. org/10.1155/2013/501350

24. Ledent C, Valverde O, Cossu G, Petitet F, Aubert JF, Beslot F, Bohme GA, Imperato A, Pedrazzini T, Roques BP et al (1999) Unresponsiveness to cannabinoids and reduced addictive effects of opiates in CB1 receptor knockout mice. Science 283:401-404. https://doi.org/10.1126/science.283.5400.401

25. Patel R, Al-Dossary AA, Stabley DL, Barone C, Galileo DS, Strehler EE, Martin-DeLeon PA (2013) Plasma membrane $\mathrm{Ca}^{2+}$-ATPase 4 in murine epididymis: secretion of splice variants in the luminal fluid and a role in sperm maturation. Biol Reprod 89:6. https://doi.org/10.1095/biolreprod.113.108712

26. Liu WM, Cao YJ, Yang YJ, Li J, Hu Z, Duan EK (2006) Tetraspanin CD9 regulates invasion during mouse embryo implantation. J Mol Endocrinol 36:121-130. https://doi.org/10.1677/jme.1.01910

27. Ragusa M, Barbagallo D, Chioccarelli T, Manfrevola F, Cobellis G, Di Pietro C, Brex D, Battaglia R, Fasano S, Ferraro B et al (2019) CircNAPEPLD is expressed in human and murine spermatozoa and physically interacts with oocyte miRNAs. RNA Biol 16:1237-1248. https://doi.org/10.1080/15476286.2019.1624469

28. Ganten D, Ruckpaul K (2005) Pseudopregnant mouse In: Encyclopedic reference of genomics and proteomics in molecular medicine. Springer, Berlin. https://doi.org/10.1007/3-540-29623-9_ 8423

29. Di Pietro C, Vento M, Ragusa M, Barbagallo D, Guglielmino MR, Maniscalchi T, Duro LR, Tomasello L, Majorana A, De 
Palma A et al (2008) Expression analysis of TFIID in single human oocytes: new potential molecular markers of oocyte quality. Reprod Biomed Online 17:338-349. https://doi.org/10.1016/ s1472-6483(10)60217-9

30. Kaufman MH (2018) Parthenogenetic activation of oocytes. Cold Spring Harb Protoc. https://doi.org/10.1101/pdb.prot094409

31. Tagliaferri D, Mazzone P, Noviello TMR, Addeo M, Angrisano T, Del Vecchio L, Visconte F, Ruggieri V, Russi S, Caivano A et al (2020) Retinoic acid induces embryonic stem cells (ESCs) transition to 2 cell-like state through a coordinated expression of Dux and Duxbl1. Front Cell Dev Biol 7:385. https://doi.org/10. 3389/fcell.2019.00385

32. Cobellis G, Cacciola G, Scarpa D, Meccariello R, Chianese R, Franzoni MF, Mackie K, Pierantoni R, Fasano S (2006) Endocannabinoid system in frog and rodent testis: type-1 cannabinoid receptor and fatty acid amide hydrolase activity in male germ cells. Biol Reprod 75:82-89. https://doi.org/10.1095/biolreprod. 106.051730

33. Nixon B, De Iuliis GN, Hart HM, Zhou W, Mathe A, Bernstein IR, Anderson AL, Stanger SJ, Skerrett-Byrne DA, Jamaluddin MFB et al (2019) Proteomic profiling of mouse epididymosomes reveals their contributions to post-testicular sperm maturation. Mol Cell Proteomics 18:S91-S108. https://doi.org/10.1074/mcp. RA118.000946

34. Hansen TB, Jensen TI, Clausen BH, Bramsen JB, Finsen B, Damgaard CK, Kjems J (2013) Natural RNA circles function as efficient microRNA sponges. Nature 495:384-388. https://doi.org/ 10.1038/nature 11993

35. Harikae K, Miura K, Kanai Y (2013) Early gonadogenesis in mammals: significance of long and narrow gonadal structure. Dev Dyn 242:330-338. https://doi.org/10.1002/dvdy.23872

36. Kuscu N, Celik-Ozenci C (2015) FOXO1, FOXO3, AND FOXO4 are differently expressed during mouse oocyte maturation and preimplantation embryo development. Gene Expr Patterns 18:16-20. https://doi.org/10.1016/j.gep.2015.04.003

37. Gordon J (2018) Hox genes in the pharyngeal region: how Hoxa3 controls early embryonic development of the pharyngeal organs. Int J Dev Biol 62:775-783. https://doi.org/10.1387/ijdb.180284jg

38. Fan J, Ponferrada VG, Sato T, Vemaraju S, Fruttiger M, Gerhardt H, Ferrara N, Lang RA (2014) Crim1 maintains retinal vascular stability during development by regulating endothelial cell Vegfa autocrine signaling. Development 141:448-459. https://doi.org/ 10.1242/dev.097949

39. Sesé B, Barrero MJ, Fabregat MC, Sander V, Izpisua Belmonte JC (2013) SMYD2 is induced during cell differentiation and participates in early development. Int J Dev Biol 57:357-364. https:// doi.org/10.1387/ijdb.130051ji

40. De Robertis EM (2009) Spemann's organizer and the self-regulation of embryonic fields. Mech Dev 126:925-941. https://doi.org/ 10.1016/j.mod.2009.08.004

41. Zalzman M, Falco G, Sharova LV, Nishiyama A, Thomas M, Lee SL, Stagg CA, Hoang HG, Yang HT, Indig FE et al (2010) Zscan4 regulates telomere elongation and genomic stability in ES cells. Nature 464:858-863. https://doi.org/10.1038/nature08882

42. Macfarlan TS, Gifford WD, Driscoll S, Lettieri K, Rowe HM, Bonanomi D, Firth A, Singer O, Trono D, Pfaff SL (2012) Embryonic stem cell potency fluctuates with endogenous retrovirus activity. Nature 487:57-63. https://doi.org/10.1038/nature11244

43. Tagliaferri D, De Angelis MT, Russo NA, Marotta M, Ceccarelli M, Del Vecchio L, De Felice M, Falco G (2016) Retinoic acid specifically enhances embryonic stem cell metastate marked by Zscan4. PLoS One 11:e0147683. https://doi.org/10.1371/journal. pone. 0147683

44. Cescon M, Chianese R, Tavares RS (2020) Environmental impact on male (In)fertility via epigenetic route. J Clin Med 9:E2520. https://doi.org/10.3390/jcm9082520
45. Tavares RS, Ramalho-Santos J (2021) The role of sperm and oocyte in fetal programming: Is Lamarck making a comeback? Eur J Clin Invest 15:e13521. https://doi.org/10.1111/eci.13521

46. Dong WW, Li HM, Qing XR, Huang DH, Li HG (2016) Identification and characterization of human testis derived circular RNAs and their existence in seminal plasma. Sci Rep 6:39080. https:// doi.org/10.1038/srep39080

47. Lin X, Han M, Cheng L, Chen J, Zhang Z, Shen T, Wang M, Wen B, Ni T, Han C (2016) Expression dynamics, relationships, and transcriptional regulations of diverse transcripts in mouse spermatogenic cells. RNA Biol 13:1011-1024. https://doi.org/ 10.1080/15476286.2016.1218588

48. Zhou T, Xie X, Li M, Shi J, Zhou JJ, Knox KS, Wang T, Chen Q, Gu W (2018) Rat BodyMap transcriptomes reveal unique circular RNA features across tissue types and developmental stages. RNA 24:1443-1456. https://doi.org/10.1261/rna.067132.118

49. Manfrevola F, Chioccarelli T, Cobellis G, Fasano S, Ferraro B, Sellitto C, Marella G, Pierantoni R, Chianese R (2020) CircRNA role and circRNA-dependent network (ceRNET) in asthenozoospermia. Front Endocrinol (Lausanne) 11:395. https://doi.org/10. 3389/fendo.2020.00395

50. Holdt LM, Kohlmaier A, Teupser D (2018) Molecular roles and function of circular RNAs in eukaryotic cells. Cell Mol Life Sci 75:1071-1098. https://doi.org/10.1007/s00018-017-2688-5

51. Conn SJ, Pillman KA, Toubia J, Conn VM, Salmanidis M, Phillips CA, Roslan S, Schreiber AW, Gregory PA, Goodall GJ (2015) The RNA binding protein quaking regulates formation of circRNAs. Cell 160:1125-1134. https://doi.org/10.1016/j.cell.2015.02.014

52. Ashwal-Fluss R, Meyer M, Pamudurti NR, Ivanov A, Bartok O, Hanan M, Evantal N, Memczak S, Rajewsky N, Kadener S (2014) CircRNA biogenesis competes with pre-mRNA splicing. Mol Cell 56:55-66. https://doi.org/10.1016/j.molcel.2014.08.019

53. Zhang Y, Xue W, Li X, Zhang J, Chen S, Zhang JL, Yang L, Chen LL (2016) The biogenesis of nascent circular RNAs. Cell Rep 15:611-624. https://doi.org/10.1016/j.celrep.2016.03.058

54. Ragan C, Goodall GJ, Shirokikh NE, Preiss T (2019) Insights into the biogenesis and potential functions of exonic circular RNA. Sci Rep 9:2048. https://doi.org/10.1038/s41598-018-37037-0

55. Kuroda M, Sok J, Webb L, Baechtold H, Urano F, Yin Y, Chung P, de Rooij DG, Akhmedov A, Ashley T et al (2000) Male sterility and enhanced radiation sensitivity in TLS(-/-) mice. EMBO J 19:453-462. https://doi.org/10.1093/emboj/19.3.453

56. Snyder EM, Licht K, Braun RE (2017) Testicular adenosine to inosine RNA editing in the mouse is mediated by ADARB1. Biol Reprod 96:244-253. https://doi.org/10.1095/biolreprod.116. 145151

57. Chianese R, Ciaramella V, Scarpa D, Fasano S, Pierantoni R, Meccariello R (2012) Anandamide regulates the expression of GnRH1, GnRH2, and GnRH-Rs in frog testis. Am J Physiol Endocrinol Metab 303:E475-E487. https://doi.org/10.1152/ajpendo. 00086.2012

58. Ciaramella V, Meccariello R, Chioccarelli T, Sirleto M, Fasano S, Pierantoni R, Chianese R (2016) Anandamide acts via kisspeptin in the regulation of testicular activity of the frog, Pelophylax esculentus. Mol Cell Endocrinol 420:75-84. https://doi.org/10.1016/j. mce.2015.11.011

59. Nielsen JE, Rolland AD, Rajpert-De Meyts E, Janfelt C, Jørgensen A, Winge SB, Kristensen DM, Juul A, Chalmel F, Jégou B et al (2019) Characterisation and localisation of the endocannabinoid system components in the adult human testis. Sci Rep 9:12866. https://doi.org/10.1038/s41598-019-49177-y

60. Howlett AC, Abood ME (2017) CB1 and CB2 receptor pharmacology. Adv Pharmacol 80:169-206. https://doi.org/10.1016/bs. apha.2017.03.007

61. Baldassano S, Serio R, Mule' F (2008) Cannabinoid CB (1) receptor activation modulates spontaneous contractile activity in mouse 
ileal longitudinal muscle. Eur J Pharmacol 582:132-138. https:// doi.org/10.1016/j.ejphar.2007.12.016

62. Ma L, Jia J, Niu W, Jiang T, Zhai Q, Yang L, Bai F, Wang Q, Xiong L (2015) Mitochondrial CB1 receptor is involved in ACEAinduced protective effects on neurons and mitochondrial functions. Sci Rep 5:12440. https://doi.org/10.1038/srep12440

63. Starke S, Jost I, Rossbach O, Schneider T, Schreiner S, Hung LH, Bindereif A (2015) Exon circularization requires canonical splice signals. Cell Rep 10:103-111. https://doi.org/10.1016/j. celrep.2014.12.002

64. Zhou W, De Iuliis GN, Dun MD, Nixon B (2018) Characteristics of the epididymal luminal environment responsible for sperm maturation and storage. Front Endocrinol (Lausanne) 9:59. https:// doi.org/10.3389/fendo.2018.00059

65. Sullivan R (2015) Epididymosomes: a heterogeneous population of microvesicles with multiple functions in sperm maturation and storage. Asian J Androl 17:726-729. https://doi.org/10.4103/ 1008-682X.155255

66. Gervasi MG, Visconti PE (2017) Molecular changes and signaling events occurring in spermatozoa during epididymal maturation. Andrology 5:204-218. https://doi.org/10.1111/andr.12320

67. Candenas L, Chianese R (2020) Exosome composition and seminal plasma proteome: a promising source of biomarkers of male infertility. Int J Mol Sci 21:7022. https://doi.org/10.3390/ijms2 1197022

68. Gagliardi M, Matarazzo MR (2016) RIP: RNA immunoprecipitation. Methods Mol Biol 1480:73-86. https://doi.org/10.1007/ 978-1-4939-6380-5_7

69. Burke KA, Janke AM, Rhine CL, Fawzi NL (2015) Residue-byresidue view of in vitro FUS granules that bind the C-terminal domain of RNA polymerase II. Mol Cell 60:231-241. https://doi. org/10.1016/j.molcel.2015.09.006

70. Saez F, Frenette G, Sullivan R (2003) Epididymosomes and prostasomes: their roles in posttesticular maturation of the sperm cells. J Androl 24:149-154. https://doi.org/10.1002/j.1939-4640.2003. tb02653.x

71. Frenette G, Sullivan R (2001) Prostasome-like particles are involved in the transfer of $\mathrm{P} 25 \mathrm{~b}$ from the bovine epididymal fluid to the sperm surface. Mol Reprod Dev 59:115-121. https://doi. org/10.1002/mrd.1013

72. Martin-DeLeon PA (2015) Epididymosomes: transfer of fertilitymodulating proteins to the sperm surface. Asian J Androl 17:720 725. https://doi.org/10.4103/1008-682X.155538

73. Zhou W, Stanger SJ, Anderson AL, Bernestein IR, De Iuliis GN, McCluskey A, McLaughlin EA, Dun MD, Nixon B (2019) Mechanisms of tethering and cargo transfer during epididymosomesperm interactions. BMC Biol 17:35. https://doi.org/10.1186/ s12915-019-0653-5

74. Wang S, Kou Z, Jing Z, Zhang Y, Guo X, Dong M, Wilmut I, Gao $S$ (2010) Proteome of mouse oocytes at different developmental stages. Proc Natl Acad Sci USA 107:17639-17644. https://doi. org/10.1073/pnas.1013185107

75. Napolitano G, Tagliaferri D, Fusco S, Cirillo C, De Martino I, Addeo M et al (2019) A novel member of prame family, Gm12794c, counteracts retinoic acid differentiation through the methyl-transferase activity of PRC2. Cell Death Differ 27:345362. https://doi.org/10.1038/s41418-019-0359-9

76. Legnini I, Di Timoteo G, Rossi F, Morlando M, Briganti F, Sthandier O, Fatica A, Santini T, Andronache A, Wade M et al (2017) Circ-ZNF609 is a circular RNA that can be translated and functions in myogenesis. Mol Cell 66:22-37.e9. https://doi.org/ 10.1016/j.molcel.2017.02.017

77. Pamudurti NR, Bartok O, Jens M, Ashwal-Fluss R, Stottmeister C, Ruhe L, Hanan M, Wyler E, Perez-Hernandez D, Ramberger E et al (2017) Translation of CircRNAs. Mol Cell 66:9-21.e7. https://doi.org/10.1016/j.molcel.2017.02.021

78. Sun P, Li G (2019) CircCode: a powerful tool for identifying circRNA coding ability. Front Genet 10:10-981. https://doi.org/10. 3389/fgene.2019.00981

Publisher's Note Springer Nature remains neutral with regard to jurisdictional claims in published maps and institutional affiliations. 